\title{
The Wightman Axioms for the Fermionic Federbush Model
}

\author{
S. N. M. Ruijsenaars ${ }^{\star}$ \\ Mathematics Department, Tübingen University, D-7400 Tübingen, Federal Republic of Germany
}

Abstract. All Wightman axioms, including asymptotic completeness, are proved for the Federbush model with coupling constants in the range $(-1 / 2,1 / 2)$.

\section{Introduction}

The relativistic quantum field theory under consideration in this paper was invented and formally solved by Federbush in 1961 [1,2]. Called the Federbush model ever since, it describes two species of one-dimensional charged massive fermions interacting through a current-pseudocurrent coupling. A few years later Wightman' [3] studied (among other things) a number of field theories known to be formally soluble, and in particular the Federbush model, with the aim of fitting these theories into the framework of axiomatic quantum field theory. He observed that a one-dimensional massive free Dirac field has a current that is the gradient of a "pseudopotential" $\sigma$, and went on to show that $\sigma$ is a local field that is not local with respect to the free field. He then indicated how the Federbush field operator might be given a rigorous meaning in terms of the object $\exp (i \pi \lambda \sigma):$. The triple dots denote vacuum subtractions, which are already necessary to ensure the object $\sigma^{n}$ has a well-defined meaning. In subsequent unpublished work Challifour and Wightman [4] proved the field $: \sigma^{n}:$ is a local operator-valued tempered distribution, but they could not show this for the field $: \exp (i \pi \lambda \sigma):$. However, in a later paper Challifour [5] did show the time-ordered Green's functions of $: \exp (i \pi \lambda \sigma)$ : exist for $|\hat{\lambda}|$ small.

Interest in the model revived in the seventies in connection with work on the massive Thirring and sine-Gordon theories. It was claimed by Tapper [6] nonzero reflection occurs in second-order renormalized perturbation theory, contradicting the absence of reflection to all orders claimed in $[1,3]$. This was subsequently refuted by several authors [7-10], who pointed out the inclusion of appropriate counterterms (needed also to respect Ward identities) does lead to vanishing reflection in second order. In the process a discrepancy between the $S$-operators of $[1,3]$ was resolved by Schroer, Truong, and Weisz, who also

* Alexander-von-Humboldt fellow 
studied operator-product expansions [10]. They used a Wick-ordered field $\varphi_{\lambda}$ that had been shown to be formally equal to $\exp (i \pi \lambda \sigma) \vdots$ by Lehmann and Stehr [11].

In a related but independent development Sato, Miwa, and Jimbo published a series of papers on what they called holonomic quantum fields [12-16]. [The term is inspired by the fact that the Schwinger functions and other associated correlation functions of these fields satisfy holonomic (integrable) systems of nonlinear and linear partial differential equations, which had been previously studied by these authors.] They were in particular interested in understanding the continuum field theory that is the scaling limit of the two-dimensional Ising model, but they also studied a bosonic analog of the Ising model and boson fields $\varphi_{\lambda}^{F}$ and $\varphi_{\lambda}^{B}$, expressed in the annihilation and creation operators on a charged fermion and boson Fock space, respectively. Only recently they realized the field $\varphi_{\lambda}^{F}$ is Wightman's field : $\exp (i \pi \lambda \sigma):$ in disguise, and established the relation of their work to the literature on the Federbush and massless Thirring models [17].

We took inspiration from their work [12-16] and our previous work on Bogoliubov transformations $[18,19]$ to begin a mathematically rigorous study of these models [20]. We introduced bosonic analogs of the Federbush and massless Thirring models, and showed that the fields of the models mentioned above are closely related or equal to quadratic forms implementing Bogoliubov transformations generated by local and covariant classical field operators, the locality and covariance of the quantum fields being a formal consequence of this. In our formalism the quantum fields appear in Wick-ordered form (in contrast to the triple-dot fields), and therefore are easily seen to give rise to matrix elements that are $C^{\infty}$ in $x \in \mathbb{R}^{2}$ (for a dense set of states). One can therefore rigorously investigate the equation of motion by letting the linear (free) part of the differential operator involved act on these $C^{\infty}$-functions. The result of this is by construction the matrix element of a normal-ordered expression. For the Federbush model on the unphysical sector (i.e., "Dirac sea unfilled") this expression is just the nonlinear term occurring at the classical level, provided that the coupling constant dependence of the solution presented in the literature is changed. If one starts from the formal solution on the physical, positive energy sector ("Dirac sea filled"), the expression has the same properties, but now one also obtains an additional factor in the free fields that occur in the expression. However, provided a similar factor in the field $\varphi_{\lambda}$ is omitted, one does get a solution to the equation of motion with the nonlinear term defined through Wick ordering [20]. The field thus obtained is most likely non-local, but leads to the same LSZ $S$-matrix as the formal solution [21].

In another paper [22] we studied the two-point functions of the above-mentioned models. We proved in particular that the Federbush field does not satisfy canonical anticommutation relations. On a formal level this can be understood from the fact that an infinite wave function renormalization is needed to define the field. However, this circumstance entails that one cannot expect that the field satisfies the equation of motion if the nonlinear terms are defined through normal ordering, a point emphasized in particular by Klauder [23]. Rather, one should define the nonlinear terms through short-distance expansions.

It is an interesting problem to make rigorous and nonperturbative sense of such expansions for the Federbush field, and to investigate the equation of motion 
in this context. Here we shall not consider this problem however, but rather study the more basic question whether the formal Federbush field really defines a quantum field in the precise sense of axiomatic field theory [24]. A superficial examination of the work of Sato, Miwa, and Jimbo (henceforth SMJ) referred to above might give the impression such questions have already been answered. This is however not the case. What has been proved by SMJ as concerns axiomatic properties is that the explicit but a priori formal expressions for the $n$-point Schwinger functions they derive have a well-defined meaning for large separations of the points involved and small values of their deformation parameters and coupling constants; the three regions involved shrink as $n$ increases, roughly speaking. Their arguments concerning locality depend on unproven assumptions, in particular that convergence holds true in much larger regions than covered by their convergence proof (cf. [15, Sect. 4.5]).

Before sketching the approach and results of this paper it is convenient to discuss related rigorous work on the continuum Ising model. Here, too, the innovative work of SMJ and of McCoy et al. [25] left open questions concerning the scaling limit convergence of the $n$-point lattice correlation functions for all distances between the $n$ points and concerning the validity of the OsterwalderSchrader axioms for the resulting continuum functions. These gaps were closed by two groups of authors in somewhat different ways. Palmer and Tracy [26] based their proof of scaling limit convergence and the O-S axioms on explicit determinantal formulas for the infinite lattice $n$-point functions. These expressions are a consequence of SMJ's astonishing product formulas, which had previously been proved by Palmer [27] in the infinite-dimensional case (SMJ's proof [12] only holds in the finite-dimensional case). The only axiomatic property left open in [26] is rotational invariance of the Schwinger functions; the authors state their formulas are not appropriate to prove this. O'Carroll and Schor independently proved scaling limit convergence and the $\mathrm{O}-\mathrm{S}$ axioms including rotational invariance [28]. In [28] only local invariance is proved. Global rotational invariance follows from recent work of Palmer and Tracy [44], in which also the SMJ analysis of "Euclidean wave functions" is rigorized. They employed an infinite series representation for the infinite lattice $n$-point functions they had previously derived from a Feynman-Kac formula $[29,30]$. Both in [28] and in [26] an essential ingredient of the proof is the reduction of temperedness of the $n$-point functions to temperedness of the two-point functions by means of correlation inequalities (due to Glimm and Jaffe [31] and Newman [32], respectively) bounding the $n$-point function by sums of products of two-point functions.

The methods used in this paper to prove all axioms for the Federbush model are quite different. We start with the quantum field as an explicitly given quadratic form on an explicit Fock space and prove the Wightman axioms instead of the equivalent $\mathrm{O}-\mathrm{S}$ axioms. No lattice approximation, finite-volume cutoff or infinite series expansions are used in the proof, and we need not invoke the reconstruction theorem, since we deal with the field directly. In the case of the Ising field theory the lattice approximation is of course eminently reasonable. As indicated above, the difficult problems in this approach are finding explicit formulas for the correlation functions and making sense of them, proving scaling limit convergence 
for all distances, temperedness of the two-point functions and rotational invariance of the resulting Schwinger functions. However, locality of the reconstructed Wightman field is a well-known consequence of the symmetry of the Schwinger functions [33], which trivially follows from convergence for all separations. In contrast, in our approach to the closely related Federbush model covariance and spectral properties are clear by inspection, but now the difficulty is in proving temperedness and locality of the field. (We shall presently discuss asymptotic completeness.) This is where a restriction on the coupling constant $\lambda$ arises: The kernels in the normal-ordered exponential only define bounded operators for $\lambda \in(-1 / 2,1 / 2)$, and for $|\lambda|>1 / 2$ the implementing property no longer holds. Correspondingly, the " $\varphi$-bounds" implying temperedness are only proven for $\lambda \in(-1 / 2,1 / 2)$ and the analyticity used to extend locality for small coupling is only shown to hold in the strip $|\operatorname{Re} \lambda|<1 / 2$.

We prove locality for small coupling by employing cutoff fields that are noncovariant bounded operators. These fields implement the Bogoliubov transformations generated by classical field operators that are approximately local, in the sense that they commute for a fixed pair $x, y \in \mathbb{R}^{2}$ with $x-y$ spacelike, provided the cutoff is small enough. As a consequence the implementers commute up to a phase factor that is a ratio of cutoff two-point functions. This ratio can be proven to equal one for zero cutoff. Through analyticity arguments we transfer the problem of proving convergence as the cutoff goes to zero to proving trace norm convergence of the kernel appearing in the pure creation part of the regularized cutoff field, and to proving bounds, uniform in the cutoff, on the blow-up of the trace norm of the kernel as the regularization parameter goes to zero. The same key points are used to prove locality of the Federbush field for $\lambda \in(-1 / 2,1 / 2)$. Here we also employ a cutoff approximately local free Dirac field that is a bounded operator, to ensure pointwise commutation relations make sense.

At this point it is appropriate to explain why the deep machinery developed by SMJ, in particular the PDE and product formulas referred to above, is hard to use as a tool to simplify and/or extend these results to arbitrary $\lambda$. The problem is that the PDE satisfied by the Schwinger functions are nonlinear, so that singularities for finite distances cannot be excluded; the product formulas are of little help in this regard, since it is difficult to make sense of them for small separations. In this connection we mention that we have proven finite-distance singularities indeed occur in the context of the bosonic Federbush model: For non-integer $\lambda>1$ the two-point Schwinger functions become singular at a distance that is an increasing function of $\lambda$ (cf. [22, Eqs. (2.66) and (2.87) and their proofs]).

Once one knows the axioms mentioned above hold true, it is obvious that the Federbush field satisfies all assumptions of the Haag-Ruelle theory [33], so that the question arises whether the axiom of asymptotic completeness can be proved. We solve this problem in the affirmative by combining our previous results on the LSZ limits of the Federbush field [21] with Hepp's extension of the Haag-Ruelle theory [34]. As a corollary it also follows that the polynomial domain is actually dense in the Fock space we started out from. The analogous results for the Ising field theory also hold true as a consequence of the axioms proved in $[26,28$, 44], Hepp's results [34] and our results on the LSZ limits of the Ising field [21]. 
This renders the Federbush and Ising models the first interacting relativistic field theories for which asymptotic completeness has been rigorously proved.

To our knowledge the Federbush model is also the first Lagrangian field theory that is renormalizable but not superrenormalizable for which the Wightman axioms have been proved. The main question left open by our results is what happens for $|\lambda| \geqq 1 / 2$. There are remarks pertaining to this (and other open problems) at several places in the main text. Here, we only note that it is easy to see the field is a well-defined operator for any $\lambda \in \mathbb{C}$ if it is smeared with test functions that are $C_{0}^{\infty}$ in momentum space (cf. Sects. $4 \mathrm{~A}$ and $5 \mathrm{~A}$ ). It also readily follows that this gives rise to Wightman distributions in $\mathscr{D}^{\prime}$ that are entire in the coupling constants. The question is therefore whether these distributions are tempered, and whether they have the locality property. Could it be that for $|\lambda|>1 / 2$ they are not in $S^{\prime}$, but still in one of Jaffe's classes of ultradistributions $[35,36]$ ? This is actually more or less suggested by our results on the two-point function $S_{\lambda}(t)$ of $\varphi_{\lambda}$ [22]: For $|\lambda| \leqq 1 / 4$ (and probably for $|\lambda| \leqq 1 / 2$ too) it behaves like $(1 / t)^{2 \lambda^{2}}$ for $t \rightarrow 0$ (up to eventual less singular terms), but for $|\lambda|>1 / 2$ it seems likely the blow-up is no longer polynomial; it is however certainly $O\left(\exp (1 / t)^{C(\lambda)}\right)$ for any $\lambda$, which entails the corresponding Wightman function may not be tempered, but still is an ultradistribution.

We conclude this introduction with a more detailed sketch of the paper. Chapter 2 has a preparatory character. We collect here the results from the classical (single-particle) theory we have occasion to use. Section 2A contains definitions of various operators and summarizes earlier results on classical field operators and conjugacy relations. In Sect. $2 \mathrm{~B}$ we collect bounds on trace norms of operators that are closely related to the classical field operators. Section $2 \mathrm{C}$ concerns the cutoff classical field operators that will only be used to prove locality for small coupling. We shall need quite detailed information on these operators and their conjugates, which is assembled in several subsections.

Chapter 3 prepares the ground as regards quadratic forms and operators on the charged fermion Fock space that will occur in the sequel. Some of the results have an independent interest and will therefore be denoted theorems. In Sect. 3A we consider the factors out of which the normal-ordered exponentials arising as implementers are built. In Sect. 3B we collect some results on implementers from $[18,19]$ and then proceed to define the cutoff quantum fields and prove the properties needed to establish locality of the Federbush field for small coupling.

The field $\varphi_{\lambda}$ is the subject of Chap. 4. It is defined in Sect. 4A, where also a number of simple properties is derived. The main results are stated in Sect. 4B. The $\varphi$-bound lemma (Lemma 4.1) and the locality lemma (Lemma 4.3) may be regarded as the technical core of the paper. Here we have occasion to use almost all of our results cited or derived in the preceding work. The proofs of these lemmas can be found in Sects. $4 \mathrm{C}$ and D together with remarks on $\varphi$-bounds in related models and on cutoff field operators, respectively. In Sect. $4 \mathrm{~B}$ we also derive more or less direct consequences, viz., temperedness (Lemma 4.2) and more generally the main result of Chap. 4 , the Wightman axioms for the field $\varphi_{\lambda}$ for $\lambda \in(-1 / 2,1 / 2)$ (Theorem 4.4). This result might be taken as a starting point to study the charged Federbush field $\psi_{\lambda}$ of Chap. 5 from the point of view of algebraic quantum field theory and the theory of charged superselection sectors. 
In Chap. 5 we finally consider the Federbush field $\psi_{\lambda}$ along the same lines as $\varphi_{\lambda}$. Section $5 \mathrm{~A}$ contains the definition and other preliminaries. In Sect. 5B we state the results: A $\psi$-bound lemma (Lemma 5.1), a lemma detailing commutation properties (Lemma 5.2), and the principal result of the paper, Theorem 5.3, stating all Wightman axioms including asymptotic completeness hold true for the field $\psi_{\lambda}$, provided $\lambda \in(-1 / 2,1 / 2)$. These results are proven in Sect. $5 \mathrm{C}$. The validity of the $\psi$-bound lemma is easily reduced to that of the corresponding Lemma 4.1 in Chap. 4, while the proof of Lemma 5.2 parallels the proof of the locality lemma spelled out in Sect. 4D. The only statement in Theorem 5.3 that then still needs proof is asymptotic completeness. As mentioned above this is shown by combining previous results of ours [21] with results of Hepp [34].

Throughout this paper we use the symbols $C, C(\lambda)$, and $C\left(\lambda, \lambda_{0}\right)$ to denote positive constants and positive continuous functions that vanish for $\lambda \rightarrow 0$ and $\lambda \rightarrow \lambda_{0}$, respectively. The domain of such functions will usually not be explicitly specified, as it will be clear from the context.

\section{Classical Theory}

\section{A. Preliminaries}

We begin with a summary of definitions, conventions and facts about conjugates that will be used throughout the paper. For more information the reader is referred to $[19,20]$. First, let us introduce some operators on the space $\breve{\mathscr{H}} \equiv L^{2}\left(d x^{1}\right)^{2}$ of two-component Dirac wave functions: The free Hamiltonian of mass 1 ,

$$
\check{H}_{0} \equiv-i \gamma^{5} \frac{d}{d x^{1}}+\gamma^{0}
$$

the momentum operator,

$$
\check{P}^{1} \equiv-i \frac{d}{d x^{1}}
$$

the charge conjugation operator,

$$
(\breve{C} f)\left(x^{1}\right) \equiv i \gamma^{5} \bar{f}\left(x^{1}\right),
$$

the parity operator,

$$
(\check{P} f)\left(x^{1}\right) \equiv \gamma^{0} f\left(-x^{1}\right),
$$

and the "kink operator,"

$$
\check{U}_{\lambda} \equiv \exp (i \pi \lambda \varepsilon(\cdot)), \quad \lambda \in \mathbb{C} .
$$

Here,

$$
\gamma^{0} \equiv\left(\begin{array}{ll}
0 & 1 \\
1 & 0
\end{array}\right), \quad \gamma^{5}=\gamma^{0} \gamma^{1} \equiv\left(\begin{array}{rr}
1 & 0 \\
0 & -1
\end{array}\right)
$$


and

$$
\varepsilon\left(x^{1}\right) \equiv\left\{\begin{aligned}
1, & x^{1}>0, \\
-1, & x^{1}<0 .
\end{aligned}\right.
$$

We employ a spectral representation of $\check{H}_{0}$ on the space $\mathscr{H} \equiv L^{2}(d \theta)^{2}$ of rapidity wave functions. This representation is generated by the unitary operator $W: \mathscr{H} \rightarrow \check{\mathscr{H}}$, defined by

$$
(W g)\left(x^{1}\right) \equiv(2 \pi)^{-1 / 2} \sum_{\delta=+,-} \int d \theta \exp \left(i \delta x^{1} \operatorname{sh} \theta\right) w_{\delta}(\theta) g_{\delta}(\theta)
$$

with inverse

$$
\left(W^{-1} f\right)_{\delta}(\theta)=(2 \pi)^{-1 / 2} \int d x^{1} \exp \left(-i \delta x^{1} \operatorname{sh} \theta\right) \bar{w}_{\delta}(\theta) \cdot f\left(x^{1}\right) .
$$

Here, $w_{\delta}(\theta)$ are the Dirac spinors

$$
w_{+}(\theta) \equiv\left(\frac{1}{2}\right)^{1 / 2}\left(e^{\frac{1}{2} \theta}, e^{-\frac{1}{2} \theta}\right), \quad w_{-}(\theta)=i\left(\frac{1}{2}\right)^{1 / 2}\left(e^{\frac{1}{2} \theta},-e^{-\frac{1}{2} \theta}\right) .
$$

Henceforth, we use the convention

$$
A \equiv W^{-1} \breve{A} W
$$

if $\check{A}$ is an operator on $\check{\mathscr{H}}$, and vice versa. With this convention,

$$
\begin{aligned}
\left(H_{0} f\right)_{\delta}(\theta) & =\delta \operatorname{ch} \theta f_{\delta}(\theta), \\
\left(P^{1} f\right)_{\delta}(\theta) & =\delta \operatorname{sh} \theta f_{\delta}(\theta), \\
(C f)_{\delta}(\theta) & =\bar{f}_{-\delta}(\theta), \\
(P f)_{\delta}(\theta) & =\delta f_{\delta}(-\theta),
\end{aligned}
$$

and $U_{\lambda}$ acts as convolution with

$$
\cos \pi \lambda \delta(\theta)+\frac{\sin \pi \lambda}{2 \pi}\left(\begin{array}{cc}
\frac{P}{\operatorname{sh} \frac{1}{2} \theta} & \frac{i}{\operatorname{ch} \frac{1}{2} \theta} \\
\frac{i}{\operatorname{ch} \frac{1}{2} \theta} & -\frac{P}{\operatorname{sh} \frac{1}{2} \theta}
\end{array}\right),
$$

where $P$ denotes the principal value. The representation of the Poincare group is most easily defined on $\mathscr{H}$. It is given by

$$
(U(a, \Lambda) f)_{\delta}(\theta) \equiv \exp \left(i \delta\left(a^{0} \operatorname{ch} \theta-a^{1} \operatorname{sh} \theta\right)\right) f_{\delta}(\theta-\alpha),
$$

where

$$
\Lambda \equiv\left(\begin{array}{ll}
\operatorname{ch} \alpha & \operatorname{sh} \alpha \\
\operatorname{sh} \alpha & \operatorname{ch} \alpha
\end{array}\right)
$$

We further introduce the notation

$$
\begin{aligned}
A^{x} & \equiv U(x, 1) A U(x, 1)^{*}, \\
q & \equiv P_{+}-P_{-}, \\
\mathscr{H}_{\delta} & \equiv P_{\delta} \mathscr{H} \\
A_{\delta \delta^{\prime}} & \equiv P_{\delta} A P_{\delta^{\prime}},
\end{aligned}
$$


where $P_{+}$and $P_{-}$are the spectral projections of $H_{0}$ onto $[1, \infty)$ and $(-\infty,-1]$, respectively, and note the commutation relations

$$
C P_{\delta}=P_{-\delta} C, \quad C U(x, 1)=U(x, 1) C .
$$

Let $U$ be a bounded (but not necessarily unitary) operator on $\mathscr{H}$ such that $U_{\ldots}$. has a bounded inverse [as an operator on $\left.L^{2}(d \theta) \simeq \mathscr{H}_{-}\right]$. Then the transformation $\varphi: U \rightarrow Z$, defined by

$$
\begin{aligned}
& Z_{++} \equiv U_{++}-U_{+-} U_{--}{ }^{-1} U_{-+}, \quad Z_{+-} \equiv U_{+-} U_{--}{ }^{-1}, \\
& Z_{-+} \equiv-U_{--}{ }^{-1} U_{-+}, \quad Z_{--} \equiv U_{--}{ }^{-1},
\end{aligned}
$$

is a bijection of the set of such operators, since it follows from its definition that $\varphi^{2}=1$. We shall refer to $Z$ as the conjugate of $U$. It follows from (2.24) by straightforward calculations that the conjugacy relation satisfies

$$
\begin{aligned}
& \varphi(U)=Z \Leftrightarrow \varphi\left(U^{x}\right)=Z^{x}, \\
& \varphi(U)=Z \Leftrightarrow \varphi\left(U^{-1}\right)=Z^{-1}, \\
& C U C=U^{-1} \Leftrightarrow C \varphi(U) C=\varphi(U),
\end{aligned}
$$

where we assumed $U$ and $Z$ have inverses in the domain of $\varphi$. We also note that if $U_{1}$ and $U_{2}$ are in the domain of $\varphi, U_{1} U_{2}$ in general is not, and if it is, one has $\varphi\left(U_{1} U_{2}\right) \neq \varphi\left(U_{1}\right) \varphi\left(U_{2}\right)$ in general. A final important property of $\varphi$ requires one more definition: An operator $A$ is called pseudo-unitary if it satisfies

$$
A^{*} q A=A q A^{*}=q .
$$

The property is that $\varphi$ transforms unitary and pseudo-unitary operators into pseudo-unitary and unitary operators, respectively. Again, this can be readily verified from (2.24).

We shall now determine the conjugate $Z_{\lambda}$ of $U_{\lambda}$. Since $U_{\lambda}$ acts as convolution with (2.16), it acts as multiplication by the matrix

$$
\frac{1}{\operatorname{ch} \pi y}\left(\begin{array}{cc}
\operatorname{ch} \pi(y+i \lambda) & i \sin \pi \lambda \\
i \sin \pi \lambda & \operatorname{ch} \pi(y-i \lambda)
\end{array}\right)
$$

after a Fourier transformation. For $\lambda$ in the strip $S$, defined by

$$
S \equiv\left\{\lambda \in \mathbb{C}|| \operatorname{Re} \lambda \mid<\frac{1}{2}\right\},
$$

$U_{\lambda-\text { - }}$ has a bounded inverse, so that (2.24) implies $Z_{\lambda}$ corresponds to multiplication by the matrix

$$
\frac{1}{\operatorname{ch} \pi(y-i \lambda)}\left(\begin{array}{cc}
\operatorname{ch} \pi y & i \sin \pi \lambda \\
-i \sin \pi \lambda & \operatorname{ch} \pi y
\end{array}\right)
$$

Thus, $Z_{\lambda}$ acts as convolution with

$$
\cos \pi \lambda \delta(\theta)+\frac{\sin \pi \lambda}{2 \pi} e^{\lambda \theta}\left(\begin{array}{cc}
\frac{P}{\operatorname{sh} \frac{1}{2} \theta} & \frac{i}{\operatorname{ch} \frac{1}{2} \theta} \\
-\frac{i}{\operatorname{ch} \frac{1}{2} \theta} & \frac{P}{\operatorname{sh} \frac{1}{2} \theta}
\end{array}\right), \quad \lambda \in S .
$$


Note that (2.31) implies $Z_{\lambda}$ is pseudo-unitary for $\lambda \in(-1 / 2,1 / 2)$, in accord with the unitarity of $U_{\lambda}$ for $\lambda \in(-1 / 2,1 / 2)$. Note also (2.31) leads to a well-defined operator for any $\lambda \in \mathbb{C}$, periodic in $\lambda$ and unbounded on the lines $\operatorname{Re} \lambda=1 / 2+\mathbb{Z}$. In contrast, one infers from (2.32) that $Z_{\lambda}$ considered as a form with domain $C_{0}^{\infty}$, extends to an entire, non-periodic form. For $|\operatorname{Re} \lambda|>1 / 2$ this form is most likely not the form of an operator, but it will naturally arise below nevertheless.

We conclude this section by introducing the classical field operator associated with the Federbush model. It is defined by

$$
\varphi_{\lambda, c l}(x) \equiv U_{\lambda}^{x}, \quad \lambda \in \mathbb{C},
$$

and has the crucial properties of covariance, i.e.,

$$
U(a, \Lambda) \varphi_{\lambda, c l}(x) U(a, \Lambda)^{*}=\varphi_{\lambda, c l}(\Lambda x+a),
$$

and locality, i.e.,

$$
\left[\varphi_{\lambda, c l}(x), \varphi_{\lambda^{\prime}, c l}(y)\right]_{-}=0, \quad(x-y)^{2}<0, \quad \forall \lambda, \lambda^{\prime} \in \mathbb{C} .
$$

\section{B. Trace Norm Estimates}

This section contains bounds on the trace norms of various operators that will occur in later chapters. Their derivation is based on the following simple fact.

Proposition. The operator $A$ on $L^{2}(d \theta)$, defined by

$$
A=F(\theta) G\left(i \partial_{\theta}\right) F(\theta) \text {, }
$$

where

$$
F(\cdot) \in L^{2} \cap L^{\infty}, \quad G(\cdot) \in L^{1} \cap L^{\infty},
$$

is trace class and satisfies

$$
\|A\|_{1} \leqq \frac{1}{2 \pi} \int d \theta|F(\theta)|^{2} \int d y|G(y)| .
$$

Proof. Since $G \in L^{1}, G^{1 / 2}\left(i \partial_{\theta}\right)$ acts as convolution with an $L^{2}$-function $g(\theta)$ that satisfies

$$
\int d \theta|g(\theta)|^{2}=\frac{1}{2 \pi} \int d y|G(y)|
$$

Hence,

$$
\begin{aligned}
\|A\|_{1} & \leqq\left\|F(\theta) G^{1 / 2}\left(i \partial_{\theta}\right)\right\|_{2}\left\|G^{1 / 2}\left(i \partial_{\theta}\right) F(\theta)\right\|_{2} \\
& =\int d \theta_{1} d \theta_{2}\left|F\left(\theta_{1}\right) g\left(\theta_{1}-\theta_{2}\right)\right|^{2} \\
& =\frac{1}{2 \pi} \int d \theta|F(\theta)|^{2} \int d y|G(y)|,
\end{aligned}
$$

concluding the proof.

The $L^{\infty}$-restriction in (2.37) can be dropped, but we do not need this. As is clear from (2.31), $Z_{\lambda+-}$ is norm-analytic in $S$. We denote its $\lambda$-derivative by $Z_{\lambda+-}^{\prime}$. We now introduce a regularization

$$
Z_{\lambda+-}^{(\prime) t} \equiv e^{-\frac{1}{2} t \operatorname{ch} \theta} Z_{\lambda+-}^{(\prime)} e^{-\frac{1}{2} t \operatorname{ch} \theta},
$$


where $\lambda \in S$ and the regularization parameter $t$ is strictly positive. We collect the bounds we need in the following lemma. As regards notation used, recall the convention made at the end of Chap. 1 . Also, $K_{0}$ denotes the modified Bessel function of zeroth order.

Lemma 2.1. The following bounds hold true for $\lambda, \lambda_{0} \in S$ :

$$
\begin{aligned}
& \left\|\exp \left(-\frac{1}{2} t \operatorname{ch} \theta\right) Z_{\lambda+-}{ }^{*} Z_{\lambda+-} \exp \left(-\frac{1}{2} t \operatorname{ch} \theta\right)\right\|_{1} \leqq C(\lambda) K_{0}(t), \\
& \left\|Z_{\lambda+-}^{t}\right\|_{1} \leqq C(\lambda) K_{0}(t), \\
& \left\|Z_{\lambda+-}^{t}-Z_{\lambda_{0}+-}^{t}\right\|_{1} \leqq C\left(\lambda, \lambda_{0}\right) K_{0}(t), \\
& \left\|\left(Z_{\lambda_{+-}}^{t}-Z_{\lambda_{0}+-}^{t}\right)\left(\lambda-\lambda_{0}\right)^{-1}-Z_{\lambda_{0}+-}^{\prime t}\right\|_{1} \leqq C\left(\lambda, \lambda_{0}\right) K_{0}(t), \\
& \left\|Z_{\lambda+-}^{t}-Z_{\lambda_{0}+-}^{t}\right\|_{1}^{2}\left|\lambda-\lambda_{0}\right|^{-1} \leqq C\left(\lambda, \lambda_{0}\right) K_{0}(t), \\
& \left\|Z_{\lambda+-}^{t}\right\|_{1} \leqq(C(\lambda)+1) K_{0}(t) .
\end{aligned}
$$

Proof. These bounds are straightforward consequences of (2.38), the well-known relation $K_{0}(t)=\int_{0}^{\infty} d \theta \exp (-t \operatorname{ch} \theta)$, and the easily verified fact that the function $\sin \pi \lambda \operatorname{sech} \pi(y-i \lambda)$ is holomorphic in the $L^{1}(d y)$-norm for $\lambda \in S$.

\section{Cutoff Classical Field Operators}

C.1. Introduction. Let $\alpha\left(x^{1}\right)$ be a real-valued odd $C^{\infty}$-function that equals 1 for $x^{1}>1$ and is monotone increasing on $(-1,1)$. In this section we study a "smearedout kink operator" defined by

$$
\check{U}_{\lambda, \varepsilon} \equiv \exp (i \pi \lambda \alpha(\cdot / \varepsilon)), \quad \lambda \in \mathbb{C}, \quad \varepsilon>0,
$$

and related operators, in particular the cutoff classical field operators

$$
\varphi_{\lambda, \varepsilon, c l}(x) \equiv U_{\lambda, \varepsilon}^{x}, \quad \lambda \in \mathbb{C}, \quad \varepsilon>0 .
$$

Note these operators are not periodic in $\lambda$, in contrast to $U_{\lambda}$ and $\varphi_{\lambda, c l}(x)$.

We close this subsection by deriving some properties of a general character. Further properties will be considered in later subsections. When possible we restrict ourselves to $U_{\lambda, \varepsilon}$, the corresponding properties of $\varphi_{\lambda, \varepsilon, c l}(x)$ being obvious from this.

Lemma 2.2. For any $\lambda \in \mathbb{C}$ one has

$$
S-\lim _{\varepsilon \rightarrow 0} U_{\lambda, \varepsilon}=U_{\lambda} .
$$

For any $\lambda \in(-1 / 4,1 / 4) U_{\lambda, \varepsilon \delta \delta}$ has a bounded inverse satisfying

$$
\left\|U_{\lambda, \varepsilon \delta \delta}{ }^{-1}\right\| \leqq(\cos \pi \lambda-|\sin \pi \lambda|)^{-1}
$$

and

$$
S-\lim _{\varepsilon \rightarrow 0} U_{\lambda, \varepsilon \delta \delta}^{-1}=U_{\lambda \delta \delta}^{-1} .
$$

The conjugate $Z_{\lambda, \varepsilon}$ of $U_{\lambda, \varepsilon}$ exists for any $\lambda \in(-1 / 4,1 / 4)$, and one has

$$
s-\lim _{\varepsilon \rightarrow 0} Z_{\lambda, \varepsilon}=Z_{\lambda}, \quad \forall \lambda \in\left(-\frac{1}{4}, \frac{1}{4}\right)
$$


and

$$
\left\|Z_{\lambda, \varepsilon \delta-\delta}\right\|<1, \quad \forall \lambda \in(-\gamma, \gamma)
$$

where

$$
\gamma \equiv \pi^{-1} \tan ^{-1}\left(\frac{1}{2}\right)
$$

Proof. The first assertion is clear. To prove the remaining ones we note that for $\lambda \in(-1,1)$ we can write

$$
\check{U}_{\lambda, \varepsilon}=\cos \pi \lambda+i \sin \pi \lambda \check{A}_{\lambda, \varepsilon},
$$

where $\check{A}_{\lambda, \varepsilon}$ acts as multiplication by a complex-valued $C^{\infty}$-function and satisfies

$$
s-\lim _{\varepsilon \rightarrow 0} \check{A}_{\lambda, \varepsilon}=\varepsilon(\cdot) \text {. }
$$

A moment's thought also shows that

$$
\left\|A_{\lambda, \varepsilon}\right\|=1, \quad \forall \lambda \in\left[-\frac{1}{2}, \frac{1}{2}\right] .
$$

Since we may write

$$
U_{\lambda, \varepsilon \delta \delta}=\cos \pi \lambda\left(1+i \tan \pi \lambda A_{\lambda, \varepsilon \delta \delta}\right),
$$

it follows $U_{\lambda, \varepsilon \delta \delta}$ has a bounded inverse for $|\lambda|<1 / 4$, given by the Neumann series

$$
U_{\lambda, \varepsilon \delta \delta}{ }^{-1}=(\cos \pi \lambda)^{-1} \sum_{n=0}^{\infty}\left(-i \tan \pi \lambda A_{\lambda, \varepsilon \delta \delta}\right)^{n} .
$$

The bound (2.51) is clear from this. Since $\check{U}_{\lambda \delta \delta}^{-1}$ has a similar representation for $|\lambda|<1 / 4$, with $\breve{A}_{\lambda, \varepsilon \delta \delta}$ replaced by $\varepsilon(\cdot)_{\delta \delta}$, (2.52) follows from (2.57) and the norm convergence of the series. The assertions concerning $Z_{\lambda, \varepsilon}$ readily follow from the definition of the conjugate [cf. (2.24)], and from what we have already proved. In particular we have

$$
\left\|Z_{\lambda, \varepsilon \delta-\delta}\right\| \leqq\left\|U_{\lambda, \varepsilon \delta-\delta}\right\|\left\|U_{\lambda, \varepsilon--^{-1}}\right\| \leqq|\sin \pi \lambda|(\cos \pi \lambda-|\sin \pi \lambda|)^{-1},
$$

implying (2.54).

C.2. Approximate Locality. The purpose of this subsection is to state and prove the following lemma, which shows the cutoff classical field operators are "approximately local."

Lemma 2.3. Given $x, y \in \mathbb{R}^{2}$ with $(x-y)^{2}<0$, one has

$$
\left[\varphi_{\lambda, \varepsilon, c l}(x), \varphi_{\lambda^{\prime}, \varepsilon, c l}(y)\right]_{-}=0, \quad \forall \lambda, \lambda^{\prime} \in \mathbb{C},
$$

for \& small enough.

Proof. It suffices to show this for $x=(a, a+\delta)$ and $y=(-a,-a-\delta)$, where $a \geqq 0$ and $\delta>0$. Also, we need only show the commutator vanishes on the subspaces $\check{\mathscr{H}}_{\text {r }}$ and $\breve{\mathscr{H}}_{l}$ of $\check{\mathscr{H}}$ for $\varepsilon$ small enough, where $\breve{\mathscr{H}}_{r} / \breve{\mathscr{H}}_{l}$ consists of the functions in $\breve{\mathscr{H}}_{\text {whose }}$ support is to the right/left of the origin. Fixing $\varepsilon \leqq \delta$ henceforth, we assert that the operators $\check{\varphi}_{\lambda, \varepsilon, c l}(x) / \check{\varphi}_{\lambda^{\prime}, \varepsilon, c l}(y)$ act as multiplication by $\exp (-i \pi \lambda) / \exp \left(i \pi \lambda^{\prime}\right)$ on $\check{\mathscr{H}}_{l} / \check{\mathscr{H}}_{r}$ (i), and that they leave $\mathscr{H}_{r} / \breve{\mathscr{H}}_{l}$ invariant (ii). From this, (2.62) readily follows, so it 
remains to verify (i) and (ii). To see (i) holds, use the fact that the "kink width" of $\breve{U}_{\lambda, \varepsilon}$ equals $2 \varepsilon$ and use the finite propagation speed of $\exp \left(i \breve{H}_{0} t\right)$. To prove (ii), note that

$$
\check{\varphi}_{\lambda, \varepsilon, c l}(x)^{*}=\check{\varphi}_{-\bar{\lambda}, \varepsilon, c l}(x), \quad \forall \lambda \in \mathbb{C}, \quad \forall x \in \mathbb{R}^{2},
$$

so that (ii) can be reduced to the already established invariance of $\check{\mathscr{H}}_{l} / \check{\mathscr{H}}_{r}$ under $\check{\varphi}_{-\bar{\lambda}, \varepsilon, c l}(x) / \check{\varphi}_{-\bar{\lambda}^{\prime}, \varepsilon, c l}(y)$.

We remark the proof shows (2.62) is valid for any $\varepsilon \in\left(0,\left(\left|x^{1}-y^{1}\right|-\left|x^{0}-y^{0}\right|\right) / 2\right]$. We shall not need this more detailed information, however.

C.3. Implementability. Since $U_{\lambda}$ is a convolution operator, its off-diagonal parts are not in the Hilbert-Schmidt class $J_{2}$. Thus the Bogoliubov transformation it generates is not unitarily implementable. The main purpose of this subsection is to show $U_{\lambda, \varepsilon}$ does have off-diagonal parts in $J_{2}$ for any $\lambda \in \mathbb{C}$, so that it generates a unitarily implementable transformation for any $\lambda \in \mathbb{R}$. In the process we shall introduce an operator $U_{\lambda, \varepsilon}^{C}$ and derive formulas that will also be used in the two following subsections.

First we recall $\breve{U}_{\lambda, \varepsilon}$ acts as multiplication by a $C^{\infty}$-function $\exp \left(i \pi \lambda \alpha\left(x^{1} / \varepsilon\right)\right)$ that equals $e^{ \pm i \pi \lambda}$ for $x^{1} \stackrel{2}{\gtrless} \pm$. In order to exploit this, we introduce a comparison operator (which is unitary for $\lambda \in \mathbb{R}$ ),

$$
\check{U}_{\lambda, \varepsilon}^{C} \equiv \cos \pi \lambda+i \sin \pi \lambda \operatorname{th} \frac{\pi}{2}\left(\frac{\cdot}{\varepsilon}-i \lambda\right), \quad \lambda \in \mathbb{C} .
$$

The point is that the difference operator

$$
\check{D}_{\lambda, \varepsilon} \equiv \check{U}_{\lambda, \varepsilon}-\check{U}_{\lambda, \varepsilon}^{C}
$$

then acts as multiplication by a function in $S(\mathbb{R})$, while the Fourier transform of the function th $\pi / 2(x-i \lambda)$ (regarded as a tempered distribution) can be found explicitly. It reads

$$
\int d x e^{-i p x} \operatorname{th} \frac{\pi}{2}(x-i \lambda)=\frac{2}{i} P \frac{e^{\lambda p}}{\operatorname{sh} p} .
$$

For $f_{1}, f_{2} \in S(\mathbb{R})^{2}$ and $|\operatorname{Re} \lambda|<1$ we then obtain using (2.8) and (2.9),

$$
\left(f_{1}, \check{U}_{\lambda, \varepsilon}^{C} f_{2}\right)=\sum_{\delta, \delta^{\prime}=+,-} \int d \theta_{1} d \theta_{2} \overline{\left(W^{-1} f_{1}\right)_{\delta}}\left(\theta_{1}\right) U_{\lambda, \varepsilon \delta \delta^{\prime}}^{C}\left(\theta_{1}, \theta_{2}\right)\left(W^{-1} f_{2}\right)_{\delta^{\prime}}\left(\theta_{2}\right),
$$

where the kernels are given by

$$
\begin{gathered}
U_{\lambda, \varepsilon \delta \delta}^{C}\left(\theta_{1}, \theta_{2}\right)=\cos \pi \lambda \delta\left(\theta_{1}-\theta_{2}\right)+\varepsilon P C_{\lambda}\left(\varepsilon \delta\left[\operatorname{sh} \theta_{1}-\operatorname{sh} \theta_{2}\right]\right) \operatorname{ch}\left(\frac{\theta_{1}+\theta_{2}}{2}\right) \\
U_{\lambda, \varepsilon \delta-\delta}^{C}\left(\theta_{1}, \theta_{2}\right)=i \varepsilon \delta C_{\lambda}\left(\varepsilon \delta\left[\operatorname{sh} \theta_{1}+\operatorname{sh} \theta_{2}\right]\right) \operatorname{sh}\left(\frac{\theta_{1}+\theta_{2}}{2}\right)
\end{gathered}
$$

and $C_{\lambda}$ is the function

$$
C_{\lambda}(p) \equiv \sin \pi \lambda e^{\lambda p} / \pi \operatorname{sh} p
$$


Similarly, we get

$$
\begin{gathered}
D_{\lambda, \varepsilon \delta \delta}\left(\theta_{1}, \theta_{2}\right)=\varepsilon D_{\lambda}\left(\varepsilon \delta\left[\operatorname{sh} \theta_{1}-\operatorname{sh} \theta_{2}\right]\right) \operatorname{ch}\left(\frac{\theta_{1}+\theta_{2}}{2}\right), \\
D_{\lambda, \varepsilon \delta-\delta}\left(\theta_{1}, \theta_{2}\right)=i \varepsilon \delta D_{\lambda}\left(\varepsilon \delta\left[\operatorname{sh} \theta_{1}+\operatorname{sh} \theta_{2}\right]\right) \operatorname{sh}\left(\frac{\theta_{1}+\theta_{2}}{2}\right),
\end{gathered}
$$

where $D_{\lambda}$ is the $S(\mathbb{R})$-function given by

$$
\begin{aligned}
D_{\lambda}(p) \equiv & \frac{1}{2 \pi} \int d x e^{-i p x}[\cos (\pi \lambda \alpha(x))-\cos \pi \lambda \\
& \left.+i\left(\sin (\pi \lambda \alpha(x))-\sin \pi \lambda \text { th } \frac{\pi}{2}(x-i \lambda)\right)\right] .
\end{aligned}
$$

In view of (2.65) we then have

$$
U_{\lambda, \varepsilon \delta \delta^{\prime}}\left(\theta_{1}, \theta_{2}\right)=U_{\lambda, \varepsilon \delta \delta^{\prime}}^{C}\left(\theta_{1}, \theta_{2}\right)+D_{\lambda, \varepsilon \delta \delta^{\prime}}\left(\theta_{1}, \theta_{2}\right) .
$$

We are now prepared for the following lemma.

Lemma 2.4. The operators $U_{\lambda, \varepsilon \delta-\delta}$ are $\|\cdot\|_{2}$-entire functions of $\lambda$.

Proof. We begin by noting that $U_{n \lambda, \varepsilon}=\left(U_{\lambda, \varepsilon}\right)^{n}$ and that the operators $U_{\lambda, \varepsilon \delta \delta}$ are norm-entire in $\lambda$, since $\breve{U}_{\lambda, \varepsilon}$ evidently has these properties. Thus we need only show $U_{\lambda, \varepsilon \delta-\delta}$ are $\|\cdot\|_{2}$-analytic for $\lambda$ in the strip $S$. To prove this we first observe that by virtue of (2.74), (2.69), (2.72) and the estimate

$$
\int d \varphi d \theta\left|K_{\lambda}(2 \varepsilon \operatorname{sh} \varphi \operatorname{ch} \theta)\right|^{2} \operatorname{sh}^{2} \varphi \leqq \mathrm{C}^{-3} \int \mathrm{d} \mathrm{pd} \theta\left|\mathrm{K}_{\lambda}(\mathrm{p})\right|^{2} \mathrm{p}^{2} / \operatorname{ch}^{3} \theta<\infty, \quad K_{\lambda}=C_{\lambda}, D_{\lambda},
$$

the operators $U_{\lambda, \varepsilon \delta-\delta}$ are in $J_{2}$ for $\lambda \in S$. We now claim that for $\lambda, \lambda_{0} \in S$ one has

$$
|p|\left|\left(K_{\lambda}(p)-K_{\lambda_{0}}(p)\right)\left(\lambda-\lambda_{0}\right)^{-1}-K_{\lambda_{0}}^{\prime}(p)\right| \leqq C\left(\lambda, \lambda_{0}\right)\left(1+p^{2}\right)^{-1}, \quad K_{\lambda}=C_{\lambda}, D_{\lambda},
$$

where the prime denotes the pointwise $\lambda$-derivative. Clearly, this estimate ensures the analyticity in $S$ we wanted to show, so it remains to prove the estimate. It clearly holds for $K_{\lambda}=C_{\lambda}$, so let us consider $K_{\lambda}=D_{\lambda}$. By virtue of (2.73) we have

$$
\begin{aligned}
& p\left(1+p^{2}\right)\left[\left(D_{\lambda}(p)-D_{\lambda_{0}}(p)\right)\left(\lambda-\lambda_{0}\right)^{-1}-D_{\lambda_{0}}^{\prime}(p)\right] \\
& \quad=\frac{1}{2 \pi i} \int d x e^{-i p x}\left(1-\partial_{x}^{2}\right)\left[\left(F(\lambda, x)-F\left(\lambda_{0}, x\right)\right)\left(\lambda-\lambda_{0}\right)^{-1}-F^{\prime}\left(\lambda_{0}, x\right)\right],
\end{aligned}
$$

where

$$
\begin{aligned}
F(\lambda, x) \equiv & -\pi \lambda \alpha^{\prime}(x) \sin (\pi \lambda \alpha(x)) \\
& +i \pi\left[\lambda \alpha^{\prime}(x) \cos (\pi \lambda \alpha(x))-\frac{1}{2} \sin \pi \lambda / \operatorname{ch}^{2} \frac{\pi}{2}(x-i \lambda)\right] .
\end{aligned}
$$

Noting $\alpha^{\prime} \in C_{0}^{\infty}$, the estimate now readily follows for $K_{\lambda}=D_{\lambda}$, too.

In the remainder of the paper we shall only need to know the off-diagonal parts are in $J_{2}$ for small real $\lambda$. We present a stronger result, since its proof is not much 
longer and since this might be useful in a further study of the cutoff quantum field operators corresponding to $U_{\lambda, \varepsilon}$ for real $\lambda$ with an eye to operator product expansions. One useful consequence is for instance that for real $\lambda U_{\lambda, \varepsilon_{-}-}$can only fail to have a bounded inverse on a discrete subset of $\mathbb{R} \backslash(-1 / 4,1 / 4)$. This follows from (2.51) and the analytic Fredholm theorem applied to

$$
U_{-\lambda, \varepsilon-+} U_{\lambda, \varepsilon+-}=1_{--}-U_{-\lambda, \varepsilon--} U_{\lambda, \varepsilon--},
$$

since the right-hand side equals $1_{--}-\left|U_{\lambda, \varepsilon--}\right|^{2}$ for real $\lambda$. Thus the conjugate (2.24) is well defined off the exceptional set. Also, since $\check{U}_{\lambda, \varepsilon}$ commutes with $\breve{C} \breve{P}$, one has

$$
C P U_{\lambda, \varepsilon_{++}}=U_{\lambda, \varepsilon_{--}} C P, \quad \forall \lambda \in \mathbb{R},
$$

where we used (2.23) and the fact $P$ commutes with $P_{\delta}$. It follows that

$$
\operatorname{dim} \operatorname{Ker} U_{\lambda, \varepsilon_{+}+}=\operatorname{dim} \operatorname{Ker} U_{\lambda, \varepsilon_{--}}, \quad \forall \lambda \in \mathbb{R} .
$$

This in turn implies that on the exceptional set the new vacuum, though it is orthogonal to the old one, still belongs to the charge-zero sector (cf. [18, Eqs. (5.1) and (5.2)]), a fact that also follows in a different way from a recent paper by Carey et al. [37]. It is an open problem whether or not the exceptional set is empty. In this connection it may be of interest to point out that a similar question arises in the external field problem, cf. Theorem 2.8 in [38] and Theorems 2.3 and 2.4 in [39]. The approach of the paper [37] just quoted might be useful in finding answers to such questions.

We finally would like to remark that in the massless case the smeared-out kink operator $\breve{U}_{\lambda, \varepsilon}$ only has off-diagonal parts in $J_{2}$ for $\lambda \in \mathbb{Z}$; equivalently, the function that defines it must be continuous on the one-point compactification of $\mathbb{R}$. This readily verified and interesting fact was first pointed out by Raina and Wanders [40] in their study of the Schwinger model (cf. also [37]).

C.4. Trace Norm Convergence of $Z_{\lambda, \varepsilon+-}^{t}$ to $Z_{\lambda+\ldots}^{t}$. In Sect. $2 \mathrm{~B}$ we have already seen that the operator $Z_{\lambda+-}^{t}$ belongs to the trace class $J_{1}$ for any $\lambda \in S$ and $t>0$. This subsection and the next one are concerned with the analogous regularization of $Z_{\lambda, \varepsilon+-}$, the operator

$$
Z_{\lambda, \varepsilon+-}^{t} \equiv e^{-\frac{1}{2} t \operatorname{ch} \theta} Z_{\lambda, \varepsilon+-} e^{-\frac{1}{2} t \operatorname{ch} \theta}, \quad \lambda \in\left(-\frac{1}{4}, \frac{1}{4}\right), \quad t>0 .
$$

In this subsection we state and prove the following lemma.

Lemma 2.5. The operator $Z_{\lambda, \varepsilon+-}^{t}$ belongs to $J_{1}$ and satisfies

$$
\lim _{\varepsilon \rightarrow 0}\left\|Z_{\lambda, \varepsilon+-}^{t}-Z_{\lambda+-}^{t}\right\|_{1}=0
$$

for any $\lambda \in(-1 / 4,1 / 4)$ and $t>0$.

Proof. Since $\check{U}_{-\lambda} \check{U}_{\lambda}=1$, we may rewrite $Z_{\lambda+-}$ as

$$
Z_{\lambda+-}=-U_{-\lambda++}{ }^{-1} U_{-\lambda+-\cdot} .
$$

Likewise,

$$
Z_{\lambda, \varepsilon+-}=-U_{-\lambda, \varepsilon++}{ }^{-1} U_{-\lambda, \varepsilon+-} .
$$


Since we have

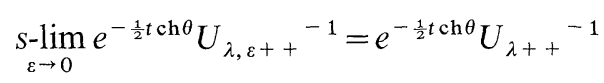

by virtue of (2.52), it suffices to prove

and

$$
\begin{gathered}
U_{\lambda+-} e^{-\frac{1}{2} t \operatorname{ch} \theta} \in J_{1}, \\
U_{\lambda, \varepsilon+-} e^{-\frac{1}{2} t \operatorname{ch} \theta} \in J_{1},
\end{gathered}
$$

$$
\lim _{\varepsilon \rightarrow 0}\left\|\left(U_{\lambda, \varepsilon+-}-U_{\lambda+-}\right) e^{-\frac{1}{2} t \operatorname{ch} \theta}\right\|_{1}=0 .
$$

To see this, one needs only verify that the assumptions $B_{\varepsilon} \rightarrow B$ strongly and $T_{\varepsilon} \rightarrow T$ in $J_{1}$ imply $B_{\varepsilon} T_{\varepsilon} \rightarrow B T$ in $J_{1}$; this easily follows by using finite-rank approximants for $T$ and the fact that $B_{\varepsilon} P \rightarrow B P$ in norm if $P$ is a finite-dimensional projection. [Note that in our case $T_{\varepsilon} B_{\varepsilon} \rightarrow T B$ in $J_{1}$ as well; the order in (2.84) and (2.85) is needed for the factorization we are about to make.]

To prove (2.87) we note $U_{\lambda+-}$ is proportional to $\operatorname{sech}\left(i \pi \partial_{\theta}\right)$ [cf. (2.29)]. Thus (2.87) holds if we show the operator $f\left(i \partial_{\theta}\right) g(\theta)$ is trace class for $f, g \in S(\mathbb{R})$. But this follows by writing it as a product of two operators that are easily seen to be in $J_{2}$ :

$$
f\left(i \partial_{\theta}\right) g(\theta)=\left[\left(\partial_{\theta}+1\right)^{-1}(\theta+i)^{-1}\right]\left[(\theta+i)\left(\partial_{\theta}+1\right) f\left(i \partial_{\theta}\right) g(\theta)\right] \text {. }
$$

We proceed to prove (2.89); from this proof and (2.87) it will be obvious (2.88) holds too. Since the operator $\left(\partial_{\theta}+1\right)^{-1} \mathrm{ch}^{-1 / 4} \theta$ is in $J_{2}$ we need only show

$$
\lim _{\varepsilon \rightarrow 0}\left\|\operatorname{ch}^{1 / 4} \theta\left(\partial_{\theta}+1\right) D_{\lambda, \varepsilon+-} e^{-\frac{1}{2} t \operatorname{ch} \theta}\right\|_{2}=0,
$$

and

$$
\lim _{\varepsilon \rightarrow 0}\left\|\operatorname{ch}^{1 / 4} \theta\left(\partial_{\theta}+1\right)\left(U_{\lambda, \varepsilon+-}^{C}-U_{\lambda+-}\right) e^{-\frac{1}{2} t \operatorname{ch} \theta}\right\|_{2}=0 .
$$

To prove (2.91) consider the kernel

$$
\varepsilon \operatorname{ch}^{1 / 4} \theta_{1}\left(\partial_{\theta_{1}}+1\right) D_{\lambda}(p) \operatorname{sh}\left(\frac{\theta_{1}+\theta_{2}}{2}\right) e^{-\frac{1}{2} t \operatorname{ch} \theta_{2}} .
$$

Here and henceforth,

$$
p \equiv \varepsilon\left(\operatorname{sh} \theta_{1}+\operatorname{sh} \theta_{2}\right) .
$$

If $\partial_{\theta_{1}}$ acts on $D_{\lambda}$ we can majorize the $L^{2}$-norm squared of the resulting kernel by

$$
\begin{aligned}
& C \varepsilon^{4} \int d \theta_{1} d \theta_{2} \operatorname{ch}^{5 / 2} \theta_{1}\left(1+p^{2}\right)^{-2} \operatorname{ch} \theta_{1} \operatorname{ch} \theta_{2} e^{-t \operatorname{ch} \theta_{2}} \\
& \quad=C \varepsilon^{3} \int d p d q\left[1+\left(\frac{p}{\varepsilon}-q\right)^{2}\right]^{5 / 4}\left(1+p^{2}\right)^{-2} \exp \left(-t\left(1+q^{2}\right)^{1 / 2}\right) \\
& \quad=O\left(\varepsilon^{1 / 2}\right) .
\end{aligned}
$$

Likewise, the remaining terms are majorized by

$$
\begin{aligned}
& C \varepsilon^{2} \int d \theta_{1} d \theta_{2} \operatorname{ch}^{1 / 2} \theta_{1}\left(1+p^{2}\right)^{-2} \operatorname{ch} \theta_{1} \operatorname{ch} \theta_{2} e^{-t \operatorname{ch} \theta_{2}} \\
& \quad=O\left(\varepsilon^{1 / 2}\right),
\end{aligned}
$$

so that (2.91) follows. 
The kernel occurring in (2.92) is proportional to

$$
\operatorname{ch}^{1 / 4} \theta_{1}\left(\partial_{\theta_{1}}+1\right) \operatorname{sech}\left(\frac{\theta_{1}-\theta_{2}}{2}\right)\left[G_{\lambda}(p)-1\right] e^{-\frac{1}{2} t \operatorname{ch} \theta_{2}},
$$

where

$$
G_{\lambda}(p) \equiv e^{\lambda p} p / \operatorname{sh} p .
$$

Consider first the term arising when $\partial_{\theta_{1}}$ acts on $G_{\lambda}$. If we get rid of the ensuing factor $\operatorname{ch}^{2} \theta_{1}$ in the $L^{2}$-norm squared of this term by using the inequality

$$
\operatorname{ch}^{2} \theta_{1}<\left(\operatorname{ch} \theta_{1}+\operatorname{ch} \theta_{2}\right)^{2} \leqq 4 \operatorname{ch}^{2}\left(\frac{\theta_{1}-\theta_{2}}{2}\right) \operatorname{ch} \theta_{1} \operatorname{ch} \theta_{2}
$$

we conclude this term is bounded by (2.96). The remaining terms can be majorized by

$$
C \int d \theta_{1} d \theta_{2} \operatorname{ch}^{1 / 2} \theta_{1} \operatorname{sech}^{2}\left(\frac{\theta_{1}-\theta_{2}}{2}\right) e^{-t \operatorname{ch} \theta_{2}}\left[G_{\lambda}(p)-1\right]^{2}
$$

Now the last term of the integrand is bounded uniformly in $\varepsilon$ and converges pointwise to zero for $\varepsilon \rightarrow 0$. But one also has

$$
\begin{aligned}
& \int d \theta_{1} d \theta_{2} \operatorname{ch}^{1 / 2} \theta_{1} \operatorname{sech}^{2}\left(\frac{\theta_{1}-\theta_{2}}{2}\right) e^{-t \operatorname{ch} \theta_{2}} \\
& \quad \leqq C \int d \varphi d \theta(\operatorname{ch} 2 \theta \operatorname{ch} \varphi)^{1 / 2} \operatorname{sech}^{2} \theta e^{-t \operatorname{ch} \varphi} \\
& \quad<\infty,
\end{aligned}
$$

so that (2.100) vanishes for $\varepsilon \rightarrow 0$ by dominated convergence. Hence, (2.92) holds true as well.

C.5. A Uniform Bound on the Trace Norm of $Z_{\lambda, \varepsilon+-}^{t}$. In this final subsection we prove $Z_{\lambda, \varepsilon+}^{t}$ - satisfies a trace norm bound similar to the bound (2.42) satisfied by $Z_{\lambda+-}^{t}$, and then add a comment on the proof and that of Lemma 2.5.

Lemma 2.6. For any $\varepsilon \in(0,1]$ and $t \in(0,1 / 4]$ one has

$$
\left\|Z_{\lambda, \varepsilon+-}^{t}\right\|_{1} \leqq C(\lambda) \ln \frac{1}{t} \text {. }
$$

Proof. We write $Z_{\lambda, \varepsilon+-}$ as $U_{\lambda, \varepsilon+-} U_{\lambda, \varepsilon_{-}-{ }^{-1}}$ and note that

$$
\left\|U_{\lambda, \varepsilon^{--}}{ }^{-1} e^{-\frac{1}{2} t \operatorname{ch} \theta}\right\| \leqq 1+C(\lambda)
$$

by virtue of (2.51). Thus we need only show

$$
\left\|e^{-\frac{1}{2} t \operatorname{ch} 0} U_{\lambda, \varepsilon+-}\right\|_{1} \leqq C(\lambda) \ln \frac{1}{t} .
$$

To this end we shall first prove that

$$
\left\|\left(\partial_{\theta}^{2}-1\right) U_{\lambda, \varepsilon+-}\right\| \leqq C(\lambda),
$$


and then that

$$
\left\|e^{-\frac{1}{2} t \operatorname{ch} \theta}\left(\partial_{\theta}^{2}-1\right)^{-1}\right\|_{1}=O\left(\ln \frac{1}{t}\right) .
$$

To prove (2.105) we note that in view of (2.56) and (2.58) one has

$$
\left\|U_{\lambda, \varepsilon+-}\right\| \leqq|\sin \pi \lambda|
$$

so that by virtue of (2.65), it suffices to show

$$
\left\|\partial_{\theta}^{2} D_{\lambda, \varepsilon+-}\right\| \leqq C(\lambda)
$$

and

$$
\left\|\partial_{\theta}^{2} U_{\lambda, \varepsilon+-}^{C}\right\| \leqq C(\lambda) .
$$

The operator in (2.108) has kernel

$$
i \varepsilon \partial_{\theta_{1}}^{2} D_{\lambda}(p) \operatorname{sh}\left(\frac{\theta_{1}+\theta_{2}}{2}\right)
$$

where $D_{\lambda}$ is defined by (2.73). [Also, recall our convention (2.94).] To prove (2.108) we shall write this kernel as a sum of kernels of integral operators whose norms we shall bound by using the well-known inequality

$$
\|I\|^{2} \leqq\left(\sup _{\theta_{1}} \int d \theta_{2}\left|K_{I}\left(\theta_{1}, \theta_{2}\right)\right|\right)\left(\sup _{\theta_{2}} \int d \theta_{1}\left|K_{I}\left(\theta_{1}, \theta_{2}\right)\right|\right) .
$$

From (2.73) it readily follows that the Fourier transform of $D_{\lambda}$ and hence $D_{\lambda}$ itself converge to zero in $S(\mathbb{R})$ for $\lambda \rightarrow 0$. We also have

$$
\left\|D_{\lambda, \varepsilon+-}\right\| \leqq 2|\sin \pi \lambda|,
$$

since the bound (2.107) also holds for $U_{\lambda . \varepsilon+-}^{C}$ by the same arguments. Thus we need only prove the assertion that for the following two types of kernels, the righthand side of (2.111) is bounded uniformly in $\varepsilon$ :

$$
\begin{aligned}
& \varepsilon^{2}\left(1+p^{2} / 4\right)^{-2} \operatorname{ch}\left(\frac{\theta_{1}+\theta_{2}}{2}\right) \operatorname{ch} \theta_{1}, \\
& \varepsilon^{3}\left(1+p^{2} / 4\right)^{-2} \operatorname{sh}\left(\frac{\theta_{1}+\theta_{2}}{2}\right) \operatorname{ch}^{2} \theta_{1} .
\end{aligned}
$$

Case (i). Using the estimate

$$
\operatorname{ch} \theta_{1} \leqq 2 \operatorname{ch}\left(\frac{\theta_{1}+\theta_{2}}{2}\right) \operatorname{ch}\left(\frac{\theta_{1}-\theta_{2}}{2}\right),
$$

we conclude it suffices to majorize the expression

$$
\begin{gathered}
\frac{1}{4} \varepsilon^{2} \sup _{\theta_{1}} \int d \theta_{2}\left(1+p^{2} / 4\right)^{-2} \operatorname{ch}^{2}\left(\frac{\theta_{1}+\theta_{2}}{2}\right) \operatorname{ch}\left(\frac{\theta_{1}-\theta_{2}}{2}\right) \\
=\varepsilon^{2} \sup _{\theta} \int_{0}^{\infty} d y\left(1+\varepsilon^{2} \operatorname{sh}^{2} y \operatorname{ch}^{2}(y-\theta)\right)^{-2} \operatorname{ch}^{2} y \operatorname{ch}(y-\theta) \\
=\varepsilon \sup _{\theta} \int_{0}^{\infty} d u\left(1+u^{2} \operatorname{ch}^{2}(y-\theta)\right)^{-2} \operatorname{ch} y \operatorname{ch}(y-\theta),
\end{gathered}
$$


where

$$
y \equiv \operatorname{sh}^{-1}(u / \varepsilon) .
$$

To this purpose we consider the integration regions $I_{1} \equiv(0, \varepsilon / 2)$ and $I_{2} \equiv(\varepsilon / 2, \infty)$ separately. The contribution of $I_{1}$ is bounded above by

$$
C \varepsilon \sup _{\theta} \int_{I_{1}} d u(1+2 u \operatorname{ch}(y-\theta))^{-2} \operatorname{ch} \theta
$$

since $\operatorname{ch} y \leqq(5 / 4)^{1 / 2}$ on $I_{1}$. Also, $\quad \mid$ th $y\left|\leqq 5^{-1 / 2}<1 / 2\right| \operatorname{cth} \theta \mid$, so that $\operatorname{ch} y \operatorname{ch} \theta \geqq 2|\operatorname{sh} y \operatorname{sh} \theta|$. Thus we have

$$
2 \operatorname{ch}(y-\theta) \geqq \operatorname{ch} y \operatorname{ch} \theta \geqq \operatorname{ch} \theta
$$

on $I_{1}$. Therefore (2.118) is bounded by

$$
\begin{aligned}
& C \varepsilon \sup _{\theta} \int_{0}^{\varepsilon / 2} d u(1+u \operatorname{ch} \theta)^{-2} \operatorname{ch} \theta=C \varepsilon \sup _{\theta}\left[-(1+u \operatorname{ch} \theta)^{-1}\right]_{0}^{\varepsilon / 2} \\
& =O(\varepsilon) .
\end{aligned}
$$

The second contribution can be bounded by

$$
\begin{aligned}
& C \int_{I_{2}} d u\left(1+u^{2}\right)^{-1}(\varepsilon / u) \operatorname{ch} y \sup _{\theta}\left[u \operatorname{ch}(y-\theta)(1+u \operatorname{ch}(y-\theta))^{-1}\right] \\
& \quad \leqq C \int_{\varepsilon / 2}^{\infty} d u\left(1+u^{2}\right)^{-1}\left[(\varepsilon / u)^{2}+1\right]^{1 / 2} \\
& \quad \leqq C .
\end{aligned}
$$

Combining this with (2.120) the assertion follows for case (i) kernels.

Case (ii). Using (2.115) we obtain as the analog of (2.116) the expression

$$
\begin{gathered}
\frac{1}{4} \varepsilon^{3} \sup _{\theta_{1}} \int d \theta_{2}\left(1+p^{2} / 4\right)^{-2}\left|\operatorname{sh}\left(\frac{\theta_{1}+\theta_{2}}{2}\right)\right| \operatorname{ch}^{2}\left(\frac{\theta_{1}+\theta_{2}}{2}\right) \operatorname{ch}^{2}\left(\frac{\theta_{1}-\theta_{2}}{2}\right) \\
=\varepsilon \sup _{\theta} \int_{0}^{\infty} d u\left(1+u^{2} \operatorname{ch}^{2}(y-\theta)\right)^{-2} u \operatorname{ch} y \operatorname{ch}^{2}(y-\theta) .
\end{gathered}
$$

Arguing as in case (i) we see this is majorized by

$$
\begin{aligned}
& C \varepsilon \sup _{\theta} \int_{0}^{\varepsilon / 2} d u\left(1+\frac{1}{4} u^{2} \operatorname{ch}^{2} \theta\right)^{-2} u \operatorname{ch}^{2} \theta \\
& \quad+C \int_{I_{2}} d u\left(1+u^{2}\right)^{-1}(\varepsilon / u) \operatorname{ch} y \sup _{\theta}\left[u^{2} \operatorname{ch}^{2}(y-\theta)\left(1+u^{2} \operatorname{ch}^{2}(y-\theta)\right)^{-1}\right] \\
& \leqq C \varepsilon \sup _{\theta}\left[-2\left(1+\frac{1}{4} u^{2} \operatorname{ch}^{2} \theta\right)^{-1}\right]_{0}^{\varepsilon / 2}+C \\
& =O(\varepsilon)+C
\end{aligned}
$$

so that the assertion follows for case (ii) kernels as well. Thus we have proven (2.108).

The operator in (2.109) has a kernel proportional to

$$
\sin \pi \lambda \partial_{\theta_{1}}^{2} G_{\lambda}(p) \operatorname{sech}\left(\frac{\theta_{1}-\theta_{2}}{2}\right),
$$


where $G_{\lambda}$ is given by (2.98). Since $\sin \pi \lambda G_{\lambda}(\cdot)$ vanishes in $S(\mathbb{R})$ for $\lambda \rightarrow 0$ and since the kernel $\operatorname{sech}\left(\left(\theta_{1}-\theta_{2}\right) / 2\right)$ defines a bounded integral operator, we need only show that for the kernels

and

$$
\varepsilon\left(1+p^{2} / 4\right)^{-2} \operatorname{ch} \theta_{1} \operatorname{sech}\left(\frac{\theta_{1}-\theta_{2}}{2}\right),
$$

$$
\varepsilon^{2}\left(1+p^{2} / 4\right)^{-2} \operatorname{ch}^{2} \theta_{1} \operatorname{sech}\left(\frac{\theta_{1}-\theta_{2}}{2}\right),
$$

the right-hand side of (2.111) is bounded uniformly in $\varepsilon$ to conclude (2.109) holds true. Using (2.115) we get the bound

$$
\begin{aligned}
& \frac{1}{4} \varepsilon \sup _{\theta_{1}} \int d \theta_{2}\left(1+p^{2} / 4\right)^{-2} \operatorname{ch}\left(\frac{\theta_{1}+\theta_{2}}{2}\right) \\
& \leqq \varepsilon \int_{0}^{\infty} d y\left(1+\varepsilon^{2} \operatorname{sh}^{2} y\right)^{-2} \operatorname{ch} y=\int_{0}^{\infty} d u\left(1+u^{2}\right)^{-2} \\
& <\infty
\end{aligned}
$$

proving this for (2.125). To prove it for (2.126) we use the estimate (2.115) to reduce this to case (i) considered above [cf. (2.113)]. Consequently, (2.109) follows and therefore (2.105) is proved.

We are now reduced to proving (2.106). To this end we cite a result of Birman and Solomjak (cf. [41, p. 55]):

$$
\left\|f(\theta) g\left(i \partial_{\theta}\right)\right\|_{1} \leqq C\|f\|_{2 ; 1}\|g\|_{2 ; 1}
$$

where

$$
\|F\|_{2 ; 1} \equiv \sum_{n=-\infty}^{\infty}\left(\int_{n}^{n+1} d \theta|F(\theta)|^{2}\right)^{1 / 2} .
$$

Thus we need only show

$$
\left\|\left(\theta^{2}+1\right)^{-1}\right\|_{2 ; 1}<\infty
$$

and

$$
\left\|e^{-\frac{1}{2} t \operatorname{ch} \theta}\right\|_{2 ; 1}=O\left(\ln \frac{1}{\mathrm{t}}\right) .
$$

Now (2.130) is obvious, while (2.131) follows once we prove

$$
\left\|\exp \left(-\frac{1}{2} e^{-N} e^{|\theta|}\right)\right\|_{2 ; 1} \leqq C N, \quad \forall N \in \mathbb{N}^{+} .
$$

But this follows from the estimates

$$
\begin{aligned}
\sum_{n=0}^{\infty}\left(\int_{n}^{n+1} d \theta \exp \left(-e^{-N+\theta}\right)\right)^{1 / 2} & \leqq N+\sum_{n=N}^{\infty}\left(\int_{\exp (-N+n)}^{\exp (-N+n+1)} \frac{d p}{p} e^{-p}\right)^{1 / 2} \\
& \leqq N+\sum_{n=0}^{\infty}\left[e^{-n} \exp \left(-e^{n}\right)\left(e^{n+1}-e^{n}\right)\right]^{1 / 2}
\end{aligned}
$$

since the series at the right-hand side converges. 
The reader may wonder whether the proofs of Lemmas 2.5 and 2.6 could not be simplified by using only one of the factorizations to prove both results. However, the factorization in Lemma 2.5 cannot lead to a bound of the form (2.102), even if $\operatorname{ch}^{1 / 4} \theta$ is replaced by $\left(1+|\theta|^{1 / 2+\delta}\right)$. The point is it would follow by taking $\varepsilon$ to 0 that

$$
\left\|\left(1+|\theta|^{1 / 2+\delta}\right)\left(\partial_{\theta}+1\right) \operatorname{sech}\left(i \pi \partial_{\theta}\right) e^{-t / 2 \operatorname{ch} \theta}\right\|_{2}
$$

is $O(\ln 1 / t)$, which is easily seen to be false. Conversely, the method of proof of Lemma 2.6 is not suitable to derive (2.83), since it is not true that $U_{\lambda, \varepsilon+-}$ converges in norm to $U_{\lambda+-}$ for $\varepsilon \rightarrow 0$. Indeed, we have seen above that $U_{\lambda, \varepsilon+-}$ is in $J_{2}$ and hence compact, while $U_{\lambda+-}$ is not compact.

\section{Fock Space Theory}

\section{A. Generalities}

In this section we collect some general properties of the special class of quadratic forms and operators on the charged fermion Fock space $\mathscr{F}_{a}(\mathscr{H}) \simeq \mathscr{F}_{a}\left(\mathscr{H}_{+}\right) \otimes \mathscr{F}_{a}\left(\mathscr{H}_{-}\right)$that will occur in the sequel. First, consider the normal-ordered exponential

$$
E(Z) \equiv: \exp \left(Z_{+-} a^{*} b^{*}+\left(Z_{++}-1\right) a^{*} a-\left(Z_{--}-1\right) b b^{*}-Z_{-+} b a\right): .
$$

Here and below $a^{(*)} / b^{(*)}$ are the particle/antiparticle creation and annihilation operators, the expression $Z a^{*} b^{*}$ (e.g.) is shorthand for the Wick monomial $\int d \theta_{1} d \theta_{2} Z\left(\theta_{1}, \theta_{2}\right) a^{*}\left(\theta_{1}\right) b^{*}\left(\theta_{2}\right)$ and the kernels define bounded operators on $L^{2}(d \theta)$. Clearly, $E(Z)$ is well defined as a quadratic form on the domain $\mathscr{D}_{\text {at }}$ of algebraic tensors, and one readily verifies that

$$
\begin{aligned}
\left\langle\psi_{1}, E(Z) \psi_{2}\right\rangle= & \left\langle\psi_{1}, \exp \left(Z_{+-} a^{*} b^{*}\right) \Gamma\left(Z_{++}\right) \Gamma\left(Z_{--}^{T}\right) \exp \left(-Z_{-+} b a\right) \psi_{2}\right\rangle \\
& \forall \psi_{1}, \psi_{2} \in \mathscr{D}_{\text {at }} .
\end{aligned}
$$

Here and henceforth we use the notation $\langle\cdot\rangle$ to denote quadratic forms and the superscript $T$ to denote the transpose. Also, $\Gamma\left(Z_{++}\right)$(e.g.) is shorthand for $\Gamma\left(Z_{++} \oplus \mathbb{1}\right) \simeq \Gamma\left(Z_{++}\right) \otimes \Gamma(\mathbb{1})$.

We proceed to study the factors at the right-hand side of (3.2) in more detail. As is well known and easily seen, the operator $\Gamma(A)$ on the boson Fock space $\mathscr{F}_{s}(\mathscr{H})$ is bounded if and only if $\|A\| \leqq 1$. On the fermion Fock space the situation is different, as shown by the following theorem.

Theorem 3.1. Let $A$ be a bounded operator on $\mathscr{H}$. Then the operator $\Gamma(A)$ on $\mathscr{F}_{a}(\mathscr{H})$ is bounded if and only if the positive part of $|A|-1$ is trace class. Furthermore,

$$
\|\Gamma(A)\|=\prod_{i=1}^{N} \lambda_{i}, \quad N \leqq \infty,
$$

where $\lambda_{1} \geqq \lambda_{2} \geqq \ldots$ are the eigenvalues of $|A|$ greater than one, counting multiplicity. 
Proof. If $\|A\| \leqq 1$ one has $\|\Gamma(A)\|=1$, so let us assume $\|A\|>1$. By the functorial property of the $\Gamma$-operation, one has $\|\Gamma(A)\|=\|\Gamma(|A|)\|$. First assume $|A|$ has some continuous spectrum in $(1,\|A\|)$. Going to a spectral representation for $|A|$, one then easily constructs a sequence of unit vectors $\varphi_{n}$ in $\mathscr{F}_{a}$ such that $\left(\varphi_{n}, \Gamma(|A|) \varphi_{n}\right) \rightarrow \infty$ for $n \rightarrow \infty$. Thus in this case $\Gamma(A)$ is unbounded. But if $|A|$ has purely discrete spectrum in $(1,\|A\|]$ it readily follows that $(3.3)$ holds. To see this, use e.g. the well-known relations $\Gamma\left(B_{1} \oplus B_{2}\right) \simeq \Gamma\left(B_{1}\right) \otimes \Gamma\left(B_{2}\right)$ and $\|C \otimes D\|$ $=\|C\|\|D\|$. Since the convergence of the product is equivalent to the trace class condition, the theorem follows.

We shall now study the form $e^{\Lambda a^{*} b^{*}}$. In the sequel we use the following convention: If $A$ is a quadratic form on $\mathscr{D}_{\text {at }}$, the phrase " $A$ is an operator" is shorthand for "The form $A$ is the form of an operator, also denoted by $A$ ".

Theorem 3.2. Let $\Lambda$ be a bounded operator on $L^{2}(d \theta)$. Then $e^{\Lambda a^{*} b^{*}}$ is an operator on $\mathscr{D}_{\text {at }}$ if and only if $\Lambda$ is Hilbert-Schmidt. Also, $e^{\Lambda a^{*} b^{*}}$ is a bounded operator if and only if $\Lambda$ is trace class. The map $\Lambda \rightarrow e^{\Lambda a^{*} b^{*}}$ is continuous from $J_{1}$ to $\mathscr{L}\left(\mathscr{F}_{a}\right)$. Moreover,

$$
\operatorname{det}\left(1+\frac{1}{2}|\Lambda|\right) \leqq\left\|e^{\Lambda a^{*} b^{*}}\right\| \leqq \operatorname{det}(1+|\Lambda|),
$$

and

$$
\left\|e^{\Lambda a^{*} b^{*}}-1\right\| \leqq \operatorname{det}(1+|\Lambda|)-1
$$

Finally,

$$
\left(e^{\Lambda_{1} a^{*} b^{*}} \Omega, e^{\Lambda_{2} a^{*} b^{*}} \Omega\right)=\operatorname{det}\left(1+\Lambda_{1}^{*} \Lambda_{2}\right), \quad \Lambda_{1}, \Lambda_{2} \in J_{2} .
$$

Proof. First assume $e^{\Lambda a^{*} b^{*}}$ is an operator on $\mathscr{D}_{\text {at }}$. Consider $\psi \in \mathscr{D}_{\text {at }}$ describing one particle and one antiparticle, so that $\psi \sim \psi\left(\theta_{1}, \theta_{2}\right)$. Since $\Omega \in \mathscr{D}_{\text {at }}$, there is a unique vector $\varphi \sim \varphi\left(\theta_{1}, \theta_{2}\right)$ such that $\left\langle\psi, e^{\Lambda a^{*} b^{*}} \Omega\right\rangle=(\psi, \varphi)$. It follows that $\varphi\left(\theta_{1}, \theta_{2}\right)$ $=\Lambda\left(\theta_{1}, \theta_{2}\right)$, so $\Lambda$ is in $J_{2}$. The converse follows from Lemma 3.1 in [18].

Now assume $e^{\Lambda a^{*} b^{*}}$ is bounded. Then $\Lambda$ is in $J_{2}$, so we can write

$$
\Lambda a^{*} b^{*}=\sum_{i=1}^{N} \lambda_{i} a^{*}\left(f_{i}\right) b^{*}\left(g_{i}\right), \quad N \leqq \infty,
$$

where $\left\{f_{i}\right\},\left\{g_{i}\right\}$ are orthonormal and where $\lambda_{i}$ are the singular values of $\Lambda$. Consider the sequence of unit vectors

$$
\varphi_{n}=\prod_{i=1}^{n} 2^{-1 / 2}\left(1+a_{i}^{*} b_{i}^{*}\right) \Omega .
$$

One has

$$
\begin{aligned}
\left\langle\varphi_{n}, e^{\Lambda a^{*} b^{*}} \varphi_{n}\right\rangle & =\left(\varphi_{n}, \exp \left(\sum_{i=1}^{n} \lambda_{i} a_{i}^{*} b_{i}^{*}\right) \varphi_{n}\right) \\
& =\prod_{i=1}^{n} \frac{1}{2}\left(\left[1+a_{i}^{*} b_{i}^{*}\right] \Omega,\left(1+\lambda_{i} a_{i}^{*} b_{i}^{*}\right)\left[1+a_{i}^{*} b_{i}^{*}\right] \Omega\right) \\
& =\prod_{i=1}^{n}\left(1+\frac{1}{2} \lambda_{i}\right),
\end{aligned}
$$



and boundedness implies the product converges for $n \rightarrow \infty$. Hence, $\sum_{i} \lambda_{i}<\infty$ so
$\Lambda$ is in $J_{1}$. Conversely, if $\Lambda$ is in $J_{1}$, one concludes from (3.7) that

$$
\left\|\Lambda a^{*} b^{*}\right\| \leqq \sum_{i=1}^{N} \lambda_{i}=\operatorname{Tr}|\Lambda|=\|\Lambda\|_{1},
$$

so that $\Lambda a^{*} b^{*}$, and hence $e^{\Lambda a^{*} b^{*}}$ too, are bounded. Also,

$$
\begin{aligned}
\left\|e^{\Lambda a^{*} b^{*}}\right\| & =\prod_{i=1}^{N}\left\|1+\lambda_{i} a_{i}^{*} b_{i}^{*}\right\| \leqq \prod_{i=1}^{N}\left(1+\lambda_{i}\right) \\
& =\operatorname{det}(1+|\Lambda|)
\end{aligned}
$$

and

$$
\begin{aligned}
\left\|e^{\Lambda a^{*} b^{*}}-1\right\| & \leqq \sum_{n=1}^{\infty} \frac{1}{n !} \sum_{i_{1}, \ldots, i_{n}=1}^{\infty} \lambda_{i_{1}} \ldots \lambda_{i_{n}}\left\|a_{i_{1}}^{*} b_{i_{1}}^{*} \ldots a_{i_{n}}^{*} b_{i_{n}}^{*}\right\| \\
= & \sum_{n=1}^{\infty} \sum_{i_{1}>i_{2} \ldots>i_{n} \geqq 1}^{\infty} \lambda_{i_{1}} \ldots \lambda_{i_{n}} \\
& =\operatorname{det}(1+|\Lambda|)-1 .
\end{aligned}
$$

The lower bound in (3.4) follows from (3.9).

Now let $\Lambda_{n} \rightarrow \Lambda$ in $J_{1}$ for $n \rightarrow \infty$. Using the bounds just proved, we then get

$$
\begin{aligned}
& \left\|e^{\Lambda a^{*} b^{*}}-e^{\Lambda a^{*} a^{*}}\right\| \leqq\left\|e^{\Lambda a^{*} b^{*}}\right\|\left\|1-e^{\left(\Lambda_{n}-\Lambda\right) a^{*} b^{*}}\right\| \\
& \leqq \operatorname{det}(1+|\Lambda|)\left[\operatorname{det}\left(1+\left|\Lambda_{n}-\Lambda\right|\right)-1\right] .
\end{aligned}
$$

Since the determinant is continuous on $J_{1}$, the right-hand side vanishes for $n \rightarrow \infty$, implying the continuity claim. Finally, (3.6) follows by making obvious changes in Eqs. (3.36)-(3.47) in [18].

In Sect. 4D we use an analyticity argument to conclude locality for $|\lambda|<1 / 2$ from locality for small coupling constant. A crucial ingredient of this argument is the following theorem and the estimate (3.14) used to prove it.

Theorem 3.3. Let $\Lambda(\lambda)$ be $\|\cdot\|_{1}$-analytic in a region $S \subset \mathbb{C}$. Then $\exp \left(\Lambda(\lambda) a^{*} b^{*}\right)$ is $\|\cdot\|$-analytic in $S$.

Proof. By virtue of Theorem 3.2 one has

$$
\begin{aligned}
&\left\|\left[\exp \left(\Lambda(\lambda) a^{*} b^{*}\right)-\exp \left(\Lambda\left(\lambda_{0}\right) a^{*} b^{*}\right)\right]\left(\lambda-\lambda_{0}\right)^{-1}-\Lambda^{\prime}\left(\lambda_{0}\right) a^{*} b^{*} \exp \left(\Lambda\left(\lambda_{0}\right) a^{*} b^{*}\right)\right\| \\
& \leqq\left.\left\|\exp \left(\Lambda\left(\lambda_{0}\right) a^{*} b^{*}\right)\right\| \|\left[\exp \left(\Lambda(\lambda)-\Lambda\left(\lambda_{0}\right)\right) a^{*} b^{*}\right)-1\right]\left(\lambda-\lambda_{0}\right)^{-1}-\Lambda^{\prime}\left(\lambda_{0}\right) a^{*} b^{*} \| \\
& \leqq \operatorname{det}\left(1+\left|\Lambda\left(\lambda_{0}\right)\right|\right)\left(\left\|\left[\left(\Lambda(\lambda)-\Lambda\left(\lambda_{0}\right)\right)\left(\lambda-\lambda_{0}\right)^{-1}-\Lambda^{\prime}\left(\lambda_{0}\right)\right] a^{*} b^{*}\right\|\right. \\
&\left.+\left|\lambda-\lambda_{0}\right|^{-1}\left\|\left[\Lambda(\lambda)-\Lambda\left(\lambda_{0}\right)\right] a^{*} b^{*}\right\|^{2} \sum_{n=2}^{\infty} \frac{1}{n !}\left\|\left[\Lambda(\lambda)-\Lambda\left(\lambda_{0}\right)\right] a^{*} b^{*}\right\|^{n-2}\right) \\
& \leqq \exp \left(\left\|\Lambda\left(\lambda_{0}\right)\right\|_{1}\right)\left[\left\|\left(\Lambda(\lambda)-\Lambda\left(\lambda_{0}\right)\right)\left(\lambda-\lambda_{0}\right)^{-1}-\Lambda^{\prime}\left(\lambda_{0}\right)\right\|_{1}\right. \\
&\left.+\left|\lambda-\lambda_{0}\right|^{-1}\left\|\Lambda(\lambda)-\Lambda\left(\lambda_{0}\right)\right\|_{1}^{2} \exp \left(\left\|\Lambda(\lambda)-\Lambda\left(\lambda_{0}\right)\right\|_{1}\right)\right] .
\end{aligned}
$$

From this the claim clearly follows. 


\section{B. Implementers and Cutoff Quantum Field Operators}

We begin this section by quoting some results from [18] which we have occasion to use. Let $U$ be a unitary operator on $\mathscr{H}$ such that $U_{\text {_. }}$ has a bounded inverse, and such that $U_{+-}$and $U_{-+}$are in $J_{2}$. Then its conjugate $Z$ defined by (2.24) is bounded and $Z_{+-}$and $Z_{-+}$are in $J_{2}$. Moreover, the quadratic form $E(Z)$ on $\mathscr{D}_{\text {at }}$ is the form of a bounded operator $E(Z)$, which satisfies

$$
E(Z) \Phi\left(U^{*} v\right)=\Phi(v) E(Z), \quad \forall v \in \mathscr{H},
$$

where $\Phi$ is the antilinear field operator

$$
\Phi(v) \equiv a\left(P_{+} v\right)+b^{*} \overline{\left(P_{-} v\right)}, \quad v \in \mathscr{H} .
$$

On $\mathscr{D}_{\text {at }}$ this operator is given by (3.1), in the sense that the expansion of the exponential strongly converges on $\mathscr{D}_{\mathrm{at}}$. The boundedness of $E(Z)$ is a consequence of the relation

$$
E(Z)=\mathscr{U} \operatorname{det}\left(1+Z_{+-}{ }^{*} Z_{+-}\right)^{1 / 2},
$$

where $\mathscr{U}$ is the unique unitary operator with positive vacuum expectation value implementing the special kind of Bogoliubov transformation to which the field operator transformation $\Phi(v) \rightarrow \Phi\left(U^{*} v\right)$ is equivalent. The adjoint of $E(Z)$ is given by

$$
E(Z)^{*}=E\left(q Z^{*} q\right),
$$

which follows, e.g., from the readily verified fact that this holds for the quadratic forms. Finally, if the Hilbert-Schmidt condition is dropped, (3.15) still holds in the sense of forms on $\mathscr{D}_{\text {at }}$. Indeed, an inspection of the proof of Theorem 4.1 in [18] shows that only the conjugacy relations (2.24) enter; the domain and convergence problems dealt with there do not arise in the form case.

Now let $U_{1}$ and $U_{2}$ be two operators with the above properties and let $E\left(Z_{1}\right)$, $E\left(Z_{2}\right)$, and $\mathscr{U}_{1}, \mathscr{U}_{2}$ be the corresponding bounded and unitary operators, respectively. Then we conclude from (3.18) and (3.6) that

$$
\left(\Omega, E\left(Z_{1}\right) E\left(Z_{2}\right) \Omega\right)=\operatorname{det}\left(1-Z_{1-+} Z_{2+-}\right) .
$$

From now on we assume in addition that $U_{1}$ and $U_{2}$ commute. Then $\mathscr{U}_{1} \mathscr{U}_{2}$ and $\mathscr{U}_{2} \mathscr{U}_{1}$ both implement the field operator transformation generated by $U_{1} U_{2}$ $=U_{2} U_{1}$, so that by uniqueness $\mathscr{U}_{1} \mathscr{U}_{2}=e^{i x} \mathscr{U}_{2} \mathscr{U}_{1}$. Taking vacuum expectation values and using (3.17) and (3.19) it follows that

$$
\operatorname{det}\left(1-Z_{1-+} Z_{2+-}\right)=e^{i \chi} \operatorname{det}\left(1-Z_{2-+} Z_{1+-}\right) .
$$

Thus we have derived the important relation

$$
E\left(Z_{1}\right) E\left(Z_{2}\right) \operatorname{det}\left(1-Z_{2-+} Z_{1+-}\right)=E\left(Z_{2}\right) E\left(Z_{1}\right) \operatorname{det}\left(1-Z_{1-+} Z_{2+-}\right) \text {. }
$$

Let us now turn to concrete operators. First, consider the unphysical Dirac representation $U(a, \Lambda)$ [cf. (2.17)]. Clearly, it is unitarily implementable, and the corresponding physical Poincaré group representation is explicitly given by

$$
\begin{aligned}
(\mathscr{U}(a, \Lambda) \psi)\left(\theta_{1}, \delta_{1} ; \ldots ; \theta_{N}, \delta_{N}\right)= & \exp \left(i a^{0} \sum_{i=1}^{N} \operatorname{ch} \theta_{i}-i a^{1} \sum_{i=1}^{N} \operatorname{sh} \theta_{i}\right) \\
& \cdot \psi\left(\theta_{1}-\alpha, \delta_{1} ; \ldots ; \theta_{N}-\alpha, \delta_{N}\right) .
\end{aligned}
$$


The energy-momentum operator $P$ is defined by

$$
e^{i a \cdot P} \equiv \mathscr{U}(a, 1) .
$$

Henceforth, we denote its zeroth component by $H$; clearly, $H$ is the secondquantized sum operator corresponding to multiplication by the function $\operatorname{ch} \theta$ on $\mathscr{H}$. For future reference we also note the relation

$$
e^{i x \cdot P} E(Z) e^{-i x \cdot P}=E\left(Z^{x}\right)
$$

[recall our convention (2.19)]. This easily follows from (3.2).

We now introduce the cutoff quantum field operators. They are defined by

$$
\varphi_{\lambda, \varepsilon}(x)=e^{i x \cdot P} E\left(Z_{\lambda, \varepsilon}\right) e^{-i x \cdot P}, \quad \lambda \in\left(-\frac{1}{4}, \frac{1}{4}\right),
$$

where $Z_{\lambda, \varepsilon}$ is the conjugate of the smeared-out kink $U_{\lambda, \varepsilon}$ (cf. Sect. $2 \mathrm{C}$ ). We collect their main properties in the following lemma.

Lemma 3.4. The field $\varphi_{\lambda, \varepsilon}(x)$ is a bounded operator satisfying

$$
\left\|\varphi_{\lambda, \varepsilon}(x)\right\|^{2}=\operatorname{det}\left(1+Z_{\lambda, \varepsilon+-} * Z_{\lambda, \varepsilon+-}\right),
$$

and implementing the transformation generated by $\varphi_{\lambda, \varepsilon, c l}(x)$. Furthermore,

$$
\varphi_{\lambda, \varepsilon}(x)^{*}=\varphi_{-\lambda, \varepsilon}(x),
$$

and

$$
\left(\Omega, \varphi_{\lambda, \varepsilon}(x) \varphi_{\lambda^{\prime}, \varepsilon}(y) \Omega\right)=\operatorname{det}\left(1-Z_{\lambda, \varepsilon-+}^{x} Z_{\lambda^{\prime}, \varepsilon+-}^{y}\right) .
$$

Finally, for $x, y \in \mathbb{R}^{2}$ with $x-y$ spacelike one has

$$
\begin{aligned}
& \varphi_{\lambda, \varepsilon}(x) \varphi_{\lambda^{\prime}, \varepsilon}(y) \operatorname{det}\left(1-Z_{\lambda^{\prime}, \varepsilon-+}^{y} Z_{\lambda, \varepsilon+-}^{x}\right) \\
& \quad=\varphi_{\lambda^{\prime}, \varepsilon}(y) \varphi_{\lambda, \varepsilon}(x) \operatorname{det}\left(1-Z_{\lambda, \varepsilon-+}^{x} Z_{\lambda^{\prime}, \varepsilon+-}^{y}\right)
\end{aligned}
$$

for $\varepsilon$ small enough.

Proof. For $x=0$ the first assertion is clear from the above and Sect. 2C.1. To see it holds for $x \neq 0$, notice (3.24) implies

$$
\varphi_{\lambda, \varepsilon}(x)=E\left(Z_{\lambda, \varepsilon}^{x}\right),
$$

and recall (2.25) and (2.49). To prove (3.27), we note first it holds for $\varphi_{\lambda, \varepsilon . c l}(x)$. Thus, if $\mathscr{U}_{\lambda, \varepsilon}(x)$ denotes the corresponding unitary implementer with positive vacuum expectation value, its inverse implements the transformation generated by $\varphi_{\lambda, \varepsilon, c l}(x)^{-1}=\varphi_{-\lambda, \varepsilon, c l}(x)$, so that $\mathscr{U}_{\lambda, \varepsilon}(x)^{*}=\mathscr{U}_{-\lambda, \varepsilon}(x)$ by uniqueness. This implies (3.27) by virtue of (3.17). Finally, (3.28) follows from (3.30) and (3.19), and (3.29) from Lemma 2.3 and (3.21).

It is understood the above statements hold for all $\lambda$ for which $\varphi_{\lambda, \varepsilon}(x)$ is defined, i.e., $\lambda \in(-1 / 4,1 / 4)$. For coupling constants in $(-\gamma, \gamma)$, where $\gamma$ is defined by (2.55), we can obtain more information on two-point functions, which is needed in Sect. 4D.

Lemma 3.5. For any $\lambda, \lambda^{\prime} \in(-\gamma, \gamma)$ and $x, y \in \mathbb{R}^{2}$ one has

$$
\left(e^{-t H} \varphi_{-\lambda, \varepsilon}(x) \Omega, e^{-t H} \varphi_{\lambda^{\prime}, \varepsilon}(y) \Omega\right) \neq 0, \quad \forall \varepsilon>0, \quad \forall t \geqq 0,
$$


and

$$
\left|\left(e^{-t H} \varphi_{-\lambda, \varepsilon}(x) \Omega, e^{-t H} \varphi_{\lambda^{\prime}, \varepsilon}(y) \Omega\right)^{-1}\right| \leqq\left(\frac{1}{t}\right)^{C\left(\lambda^{\prime}\right)}, \quad \forall \varepsilon \in(0,1], \quad \forall t \in\left(0, \frac{1}{4}\right] .
$$

Moreover, for $x, y \in \mathbb{R}^{2}$ with $x-y$ spacelike one has

$$
\begin{aligned}
& \varphi_{\lambda, \varepsilon}(x) \varphi_{\lambda^{\prime}, \varepsilon}(y)\left(\varphi_{-\lambda, \varepsilon}(x) \Omega, \varphi_{\lambda^{\prime}, \varepsilon}(y) \Omega\right)^{-1} \\
& \quad=\varphi_{\lambda^{\prime}, \varepsilon}(y) \varphi_{\lambda, \varepsilon}(x)\left(\varphi_{-\lambda^{\prime}, \varepsilon}(y) \Omega, \varphi_{\lambda, \varepsilon}(x) \Omega\right)^{-1}, \quad \forall \lambda, \lambda^{\prime} \in(-\gamma, \gamma)
\end{aligned}
$$

for \& small enough.

Proof. We first observe that

$$
\left(\varphi_{-\lambda, \varepsilon}(x) \Omega, e^{-\frac{1}{2} t H} \varphi_{\lambda^{\prime}, \varepsilon}(y) \Omega\right)=\operatorname{det}\left(1-Z_{\lambda, \varepsilon_{-}}^{x} U(y, 1)_{++} Z_{\lambda^{\prime}, \varepsilon_{+}-}^{t} U(y, 1)_{-}^{*}\right),
$$

where we used (3.6). We now recall the formula

$$
\operatorname{det}(1+T)=\prod_{i}\left(1+\lambda_{i}\right)
$$

where $\lambda_{i}$ are the eigenvalues of $T$. Thus the determinant cannot vanish if $\|T\|<1$. From (2.54) and (3.34) it therefore follows that (3.31) holds true. The relation (3.33) is now obtained from (3.29) by division, which is allowed in view of (3.31). Finally, if $\|T\|<1$, it is easy to see that

$$
|\operatorname{det}(1+T)|^{-1} \leqq \exp \left(\|T\|_{1}(1-\|T\|)^{-1}\right) .
$$

Combining this with (3.34) and (2.102), (3.32) follows.

The relation (3.33) is one of the cornerstones of the locality proofs below. Some simplification would occur if the two vacuum expectation values would be equal and hence could be omitted. Proving or disproving this would also be of interest, since the cutoff fields can probably be used to study short-distance expansions. To explain what is involved here, let us first return to the general situation discussed at the beginning of this section [cf. (3.19)-(3.21)]. The conjugacy relations (2.24) imply that

$$
\operatorname{det}\left(1-Z_{1-+} Z_{2+-}\right)=\operatorname{det}\left(U_{1--^{-1}}\left(U_{1} U_{2}\right)_{--} U_{2--}{ }^{-1}\right) .
$$

This shows, first, that vanishing of the determinant implies $\left(U_{1} U_{2}\right)_{-}$does not have a bounded inverse; using the Fredholm alternative one sees the converse is true, too. Second, it shows that if $U_{1}$ and $U_{2}$ commute, so that (3.20) holds, and if in addition the determinants are non-zero, the phase factor $e^{i \chi}$ is a quotient of $\operatorname{det} A B C$ and $\operatorname{det} C B A$, where $A, B$, and $C$ are bounded operators with bounded inverses, such that the products $A B C$ and $C B A$ equal the identity plus a trace class operator (abbreviated: $1+T$ ). If one of $A, B, C$ equals $1+T$, it readily follows that $e^{i \chi}=1$. (Note that in our case the unitarity of $U_{1}$ and $U_{2}$ implies $|A|,|B|$, and $|C|$ are $1+T$, so the unitary operator in the polar decomposition must be $1+T$ for this to be true.) But if this condition is violated, there seems to be no reason why the two determinants should be equal, though we do not know an explicit example where $e^{i \chi} \neq 1$. 
Returning now to the concrete operators $U_{\lambda, \varepsilon}$, we know from Sect. 2 C. 3 that their off-diagonal parts are Hilbert-Schmidt. If the inverses of the diagonal parts were $1+T$, this would hold for the diagonal parts as well. But then it would follow that $\check{U}_{\lambda, \varepsilon}-1$ is compact, which is clearly false. Thus in our case $A$ and $C$ (and probably $B$ as well) are not $1+T$, so that this avenue is closed. Let us make one more remark on the problem, however. From the relation (2.27) and the definition of $\check{U}_{\lambda, \varepsilon}$, it follows that $Z_{\lambda, \varepsilon}$ commutes with $C$. But this is easily seen to imply equality of the determinants is equivalent to their being real-valued. [Recall (2.23) and the equality $\operatorname{det}(1-A B)=\operatorname{det}(1-B A)$.] It is not hard to see that reality does hold for $x^{0}=y^{0}$, and we have some indication this may be true for $x-y$ spacelike and $\varepsilon$ small enough to guarantee commutativity, but we were unable to prove this so far.

We conclude this chapter by introducing a cutoff free Dirac field $\psi_{0, \varepsilon}$, and by establishing its commutation relations. It is defined by

$$
\psi_{0, \varepsilon}(x) \equiv e^{i x \cdot P} \psi_{0, \varepsilon}(0) e^{-i x \cdot P},
$$

where

$$
\psi_{0, \varepsilon, u}(0) \equiv \Phi\left(W^{-1}\left(\begin{array}{c}
j_{\varepsilon} \\
0
\end{array}\right)\right), \quad \psi_{0, \varepsilon, l}(0) \equiv \Phi\left(W^{-1}\left(\begin{array}{c}
0 \\
j_{\varepsilon}
\end{array}\right)\right)
$$

[cf. (2.9) and (3.16)]. Here $u / l$ denotes the upper/lower component and $j_{\varepsilon}\left(x^{1}\right) \equiv \varepsilon^{-1} j\left(x^{1} / \varepsilon\right)$ is an approximate identity. It is easily verified this implies

$$
\psi_{0, \varepsilon}(x)=\int d y^{1} j_{\varepsilon}\left(x^{1}-y^{1}\right) \psi_{0}\left(x^{0}, y^{1}\right) .
$$

Here, $\psi_{0}$ is the free Dirac field of mass 1 ,

$$
\psi_{0}(x) \equiv(4 \pi)^{-1 / 2} \int d \theta\left[a(\theta)\left(\begin{array}{c}
e^{\frac{1}{2} \theta} \\
e^{-\frac{1}{2} \theta}
\end{array}\right) e^{-i x \cdot p}+b^{*}(\theta)\left(\begin{array}{c}
i e^{\frac{1}{2} \theta} \\
-i e^{-\frac{1}{2} \theta}
\end{array}\right) e^{i x \cdot p}\right]
$$

where

$$
p \equiv(\operatorname{ch} \theta, \operatorname{sh} \theta) .
$$

Lemma 3.6. The components of $\psi_{0, \varepsilon}(x)$ are bounded operators satisfying

$$
\left\|\psi_{0, \varepsilon, i}(x)\right\|^{2}=\varepsilon^{-1} \int d x^{1} j\left(x^{1}\right)^{2}, \quad i=u, l .
$$

For $x, y \in \mathbb{R}^{2}$ with $x-y$ spacelike one has

$$
\left[\psi_{0, \varepsilon}(x), \psi_{0, \varepsilon}(y)^{*}\right]_{+}=0,
$$

and

$$
\psi_{0, \varepsilon}(x) \varphi_{\lambda, \varepsilon}(y)=\exp \left[i \pi \lambda \varepsilon\left(x^{1}-y^{1}\right)\right] \varphi_{\lambda, \varepsilon}(y) \psi_{0, \varepsilon}(x)
$$

for $\varepsilon$ small enough.

Proof. The first statement follows by recalling $W$ is unitary and noting that

$$
\|\Phi(u)\|=\|u\|, \quad \forall u \in \mathscr{H},
$$

which readily follows from the commutation relation

$$
\left[\Phi(u), \Phi(v)^{*}\right]_{+}=(u, v), \quad \forall u, v \in \mathscr{H} .
$$


The relation (3.44) follows, e.g., from (3.38), (3.39), (3.47) and the finite propagation speed of $\exp \left(i \check{H}_{0} t\right)$.

We shall now prove (3.45) for the upper component. First we note that the implementing property of $\varphi_{\lambda, \varepsilon}(y)$ implies

$$
\begin{aligned}
\psi_{0, \varepsilon, u}(x) \varphi_{\lambda, \varepsilon}(y) & =\Phi\left(W^{-1} \check{U}(x, 1)\left(\begin{array}{c}
j_{\varepsilon} \\
0
\end{array}\right)\right) E\left(Z_{\lambda, \varepsilon}^{y}\right) \\
& =E\left(Z_{\lambda, \varepsilon}^{y}\right) \Phi\left(U_{\lambda, \varepsilon}^{y}{ }^{*} W^{-1} \check{U}(x, 1)\left(\begin{array}{c}
j_{\varepsilon} \\
0
\end{array}\right)\right) \\
& =\varphi_{\lambda, \varepsilon}(y) \Phi\left(U(x, 1) W^{-1} \check{U}(y-x, 1) \check{U}_{\lambda, \varepsilon}^{*} \check{U}(y-x, 1) *\left(\begin{array}{c}
j_{\varepsilon} \\
0
\end{array}\right)\right) .
\end{aligned}
$$

By virtue of the definitions of $\check{U}_{\lambda, \varepsilon}$ and $j_{\varepsilon}$ and the finite propagation speed of $\exp \left(i \check{H}_{0} t\right)$, it now follows that

$$
\check{U}(y-x, 1) \check{U}_{\lambda, \varepsilon} * \check{U}(y-x, 1) *\left(\begin{array}{c}
j_{\varepsilon} \\
0
\end{array}\right)=\exp \left[-i \pi \lambda \varepsilon\left(x^{1}-y^{1}\right)\right]\left(\begin{array}{c}
j_{\varepsilon} \\
0
\end{array}\right),
$$

provided $\varepsilon$ is small enough. From this, (3.45) follows for the upper component. The proof for the lower component is the same.

\section{The Field $\varphi_{\lambda}$}

\section{A. Preliminaries}

From Sects. $2 \mathrm{~A}$ and $3 \mathrm{~A}$ it follows that for $\lambda \in(-1 / 2,1 / 2)$ the Bogoliubov transformation generated by the classical field operator $\varphi_{\lambda, c l}(x)$ can be implemented in $\mathscr{F}{ }_{a}(\mathscr{H})$ in the form sense. In this chapter we study the implementing quadratic form on the domain of algebraic tensors $\mathscr{D}_{\text {at }}$, the quantum field

$$
\begin{aligned}
\varphi_{\lambda}(x) & \equiv e^{i x \cdot P} E\left(Z_{\lambda}\right) e^{-i x \cdot P} \\
& =\exp \left(Z_{\lambda+-}^{x} a^{*} b^{*}+\left(Z_{\lambda++}^{x}-1\right) a^{*} a-\left(Z_{\lambda--}^{x}-1\right) b b^{*}-Z_{\lambda-+}^{x} b a\right): .
\end{aligned}
$$

For convenience we repeat the definition of the kernels:

$$
Z_{\lambda \delta \delta^{\prime}}^{x}\left(\theta_{1}, \theta_{2}\right) \equiv \exp \left(i x \cdot\left[\delta p\left(\theta_{1}\right)-\delta^{\prime} p\left(\theta_{2}\right)\right]\right) Z_{\lambda \delta \delta^{\prime}}\left(\theta_{1}-\theta_{2}\right),
$$

where

$$
Z_{\lambda}(\theta) \equiv \cos \pi \lambda \delta(\theta)+\frac{\sin \pi \lambda}{2 \pi} e^{\lambda \theta}\left(\begin{array}{cc}
\frac{P}{\operatorname{sh} \frac{1}{2} \theta} & \frac{i}{\operatorname{ch} \frac{1}{2} \theta} \\
\frac{-i}{\operatorname{ch} \frac{1}{2} \theta} & \frac{P}{\operatorname{sh} \frac{1}{2} \theta}
\end{array}\right)
$$

and

$$
p(\theta) \equiv(\operatorname{ch} \theta, \operatorname{sh} \theta)
$$


The implementing property of $\varphi_{\lambda}(x)$ is readily seen to imply the relations

and

$$
\varphi_{\lambda}(x)^{*}=\varphi_{-\lambda}(x),
$$

$$
\mathscr{U}(a, \Lambda) \varphi_{\lambda}(x) \mathscr{U}(a, \Lambda)^{*}=\varphi_{\lambda}(\Lambda x+a),
$$

where $\mathscr{U}(a, \Lambda)$ is the Poincare group representation (3.22). Both relations hold in the sense of forms on $\mathscr{D}_{\text {at }}$ and can also be easily checked directly.

We proceed by noting the function

$$
E_{\lambda, \psi_{1}, \psi_{2}}(x) \equiv\left\langle\psi_{1}, \varphi_{\lambda}(x) \psi_{2}\right\rangle, \quad \lambda \in\left(-\frac{1}{2}, \frac{1}{2}\right), \quad \psi_{1}, \psi_{2} \in \mathscr{D}_{\text {at }},
$$

is uniformly bounded and continuous on $\mathbb{R}^{2}$. From Sect. $2 \mathrm{~A}$ we also conclude it has an analytic continuation to the strip $S$ [defined by (2.30)] with the same properties. It easily follows the quadratic form $\varphi_{\lambda}(F)$ on $\mathscr{D}_{\text {at }}$, defined by

$$
\left\langle\psi_{1}, \varphi_{\lambda}(F) \psi_{2}\right\rangle=\int d x F(x) E_{\lambda, \psi_{1}, \psi_{2}}(x), \quad F \in S\left(\mathbb{R}^{2}\right), \quad \lambda \in S,
$$

is continuous on $S\left(\mathbb{R}^{2}\right)$ and analytic in $S$. To study $\varphi_{\lambda}(F)$ in more detail we introduce the dense subspace

$$
\mathscr{D}_{\infty}=\bigcup_{N=1}^{\infty} \mathscr{D}_{N},
$$

where

$$
\mathscr{D}_{N} \equiv P_{N} \mathscr{F}_{a}(\mathscr{H})
$$

and $P_{N}$ is the spectral projection of $H$ on the interval $[0, N)$. Thus $\mathscr{D}_{1}$ consists of multiples of $\Omega$, and $\mathscr{D}_{N}$ only contains wave functions describing fewer than $N$ charged fermions, whose rapidities can only vary over a bounded set (recall the rest mass equals 1 by assumption).

Consider first the form $P_{N} G_{\lambda}(x) P_{M}$ on $\mathscr{D}_{\text {at }}$ for $|\lambda|<1 / 2$, where $G_{\lambda}(x)$ is a generic term in the expansion of the exponential (4.1). We claim this form is the form of a bounded operator that admits a norm-analytic extension in $\lambda$ to $\mathbb{C}$; moreover, the resulting operator is norm continuous in $x$ and has an $x$-independent norm for fixed $\lambda \in \mathbb{C}$. To see this, first observe that the kernels $Z_{\lambda \delta \delta^{\prime}}\left(\theta_{1}-\theta_{2}\right)$ define operators on $L^{2}((-C, C), d \theta)$ with corresponding properties as long as $C<\infty$; note in particular the off-diagonal parts are $\|\cdot\|_{2}$-entire in this case. Now recall the properties of $\mathscr{D}_{N}$ and $\mathscr{D}_{M}$ and the relation $\|A \otimes B\|=\|A\|\|B\|$. Finally, note the $x$-dependence is carried by the unitary group $\exp (i x \cdot P)$. As a consequence the form $P_{N} \varphi_{\lambda}(x) P_{M}$ is (the form of) a bounded operator with the same properties. (Indeed, only a finite number of terms in the expansion contributes, viz., those with fewer than $N$ creators and $M$ annihilators.) It follows from this that for fixed $F \in S\left(\mathbb{R}^{2}\right) P_{N} \varphi_{\lambda}(F) P_{M}$ is a bounded operator with a norm-analytic extension to $\mathbb{C}$. Furthermore, the map $F \rightarrow P_{N} \varphi_{\lambda}(F) P_{M}$ is continuous from $S\left(\mathbb{R}^{2}\right)$ to $\mathscr{L}\left(\mathscr{F}_{a}\right)$.

We return to the generic term $G_{\lambda}(x)$ in the expansion of $\varphi_{\lambda}(x)$. If it has $k$ creators and $l$ annihilators, its $x$-dependence is given by a factor of the form

$$
\exp \left(i x \cdot\left[\sum_{j=1}^{k} p\left(\theta_{j}\right)-\sum_{j=k+1}^{k+l} p\left(\theta_{j}\right)\right]\right) \text {. }
$$


Now fix $F \in S\left(\mathbb{R}^{2}\right)$ whose Fourier transform $\hat{F}$ has compact support, and consider the contribution of the generic term to the form $\varphi_{\lambda}(F) P_{M}$. The second sum in (4.11) then only varies over an $M$-dependent bounded region in $\mathbb{R}^{2}$. Since the difference of the two sums varies over supp $\hat{F}$, it follows that the first sum only varies over a bounded region in $\mathbb{R}^{2}$ determined by $M$ and $\operatorname{supp} \hat{F}$. Consequently, its zeroth component is bounded above by some $N=N(M, \operatorname{supp} \hat{F})<\infty$, so that

$$
\varphi_{\lambda}(F) P_{M}=P_{N} \varphi_{\lambda}(F) P_{M} .
$$

From the previous paragraph we then conclude $\varphi_{\lambda}(F) P_{M}$ is a bounded operator that is norm-entire in $\lambda$. It also readily follows from $(4.12)$ that $\varphi_{\lambda}(F)$ is a welldefined operator on $\mathscr{D}_{\infty}$, which leaves $\mathscr{D}_{\infty}$ invariant. Furthermore, from (4.5) and (4.6) one infers that

$$
\varphi_{\lambda}(F)^{*} \uparrow \mathscr{D}_{\infty}=\varphi_{-\bar{\lambda}}(\bar{F})
$$

and

$$
\mathscr{U}(a, \Lambda) \varphi_{\lambda}(F) \mathscr{U}(a, \Lambda)^{*}=\varphi_{\lambda}\left(F^{a, \Lambda}\right),
$$

where

$$
F^{a, \Lambda}(x) \equiv F\left(\Lambda^{-1}(x-a)\right) .
$$

As another useful consequence of (4.12) we point out that if $\hat{F}_{n} \rightarrow \hat{F}$ in $\mathscr{D}\left(\mathbb{R}^{2}\right)$, it follows that $\varphi_{\lambda}\left(F_{n}\right) P_{M} \rightarrow \varphi_{\lambda}(F) P_{M}$ in norm. Finally, consider the expression

$$
\prod_{i=1}^{N} \varphi_{\lambda_{i}}\left(F_{i}\right) \Omega, \quad \hat{F}_{i} \in C_{0}^{\infty}\left(\mathbb{R}^{2}\right), \quad \lambda_{i} \in \mathbb{C}, \quad i=1, \ldots, N .
$$

Here and henceforth the order of the product is the natural order of the indices. Clearly, it follows from the above that this expression has a well-defined meaning as a vector in $\mathscr{D}_{\infty}$, which depends continuously on $\hat{F}_{i} \in \mathscr{D}\left(\mathbb{R}^{2}\right)$ and which is entire in the coupling constants $\lambda_{i}$.

\section{B. The Results}

With these preliminary observations out of the way, we are now in a position to state the main results of this chapter.

Lemma 4.1 (A $\varphi$-bound). For any $\lambda \in(-1 / 2,1 / 2)$ and $t, u>0$ the form $e^{-t H} \varphi_{\lambda}(0) e^{-u H}$ on $\mathscr{D}_{\text {at }}$ is the form of a bounded operator, satisfying

$$
\left\|e^{-t H} \varphi_{\lambda}(0) e^{-u H}\right\|=O\left(\left(\frac{1}{t u}\right)^{C(\lambda)}\right), \quad t, u \rightarrow 0 .
$$

We shall prove this lemma in Sect. 4C. Here, we draw some conclusions and prove in particular a corollary of the lemma. First, we note that the product of the three bounded operators $e^{-t_{1} H}\left(e^{-t_{2} H} \varphi_{\lambda}(0) e^{-u_{1} H}\right) e^{-u_{2} H}$ equals the operator $e^{-\left(t_{1}+t_{2}\right) H} \varphi_{\lambda}(0) e^{-\left(u_{1}+u_{2}\right) H}$, since these operators are equal as forms on $\mathscr{D}_{\text {at }}$. It follows from this that the range of $e^{-t H} \varphi_{\lambda}(0) e^{-u H}$ is contained in the domain of $e^{a H}$ for any $a<t$. This in turn implies the operator $\left(e^{-r H} \varphi_{\lambda}(0) e^{-s H}\right)\left(e^{-t H} \varphi_{\lambda^{\prime}}(0)^{-u H}\right)$ is a function of $s+t$, and can therefore be written as $e^{-r H} \varphi_{\lambda}(0) e^{-(s+t) H} \varphi_{\lambda^{\prime}}(0) e^{-u H}$ without 
ambiguity. Also, if $\chi$ is in the domain of $e^{v H}$, the vector $\left(e^{-t H} \varphi_{\lambda}(0) e^{-u H}\right) e^{u H} \chi$, where $u<v$, is independent of $u$, and may therefore be denoted $e^{-t H} \varphi_{\lambda}(0) \chi$. Of course, similar remarks apply to the bounded operator $e^{-t H} \varphi_{\lambda}(x) e^{-u H}=e^{i x \cdot P}\left(e^{-t H} \varphi_{\lambda}(0)\right.$ $\left.e^{-u H}\right) e^{-i x \cdot P}$; note, moreover, it is strongly continuous in $x$ and has an $x$-independent norm. More generally, one may replace the factors $e^{-t H}$ by $e^{-\eta \cdot P}$, where $\eta$ is in the forward light cone; note that

$$
\left\|e^{-\eta_{1} \cdot P} \varphi_{\lambda}(0) e^{-\eta_{2} \cdot P}\right\| \leqq\left\|\exp \left(-\left[\eta_{1}^{0}-\left|\eta_{1}^{1}\right|\right] H\right) \varphi_{\lambda}(0) \exp \left(-\left[\eta_{2}^{0}-\left|\eta_{2}^{1}\right|\right] H\right)\right\| .
$$

In the sequel we shall use the notation just introduced without comment, in particular in the proof of the following lemma.

Lemma 4.2 (Temperedness). Let $\lambda_{i} \in(-1 / 2,1 / 2)$ and $\hat{F}_{i} \in \mathscr{D}\left(\mathbb{R}^{2}\right), i=1, \ldots, n$. Then the distribution in $\mathscr{D}^{\prime}\left(\mathbb{R}^{2 n}\right)$ defined by

$$
\hat{\mathscr{W}}\left(\hat{F}_{1}, \ldots, \hat{F}_{n}\right) \equiv\left(\Omega, \prod_{i=1}^{n} \varphi_{\lambda_{i}}\left(F_{i}\right) \Omega\right)
$$

is the restriction of a distribution in $S^{\prime}\left(\mathbb{R}^{2 n}\right)$.

Proof. By virtue of the first statement of Lemma 4.1 and the remarks made above, the function

$$
\begin{aligned}
W\left(\xi_{1}-i \eta_{1}, \ldots, \xi_{n-1}-i \eta_{n-1}\right) \equiv & \left(\Omega, e^{-\varepsilon H} \varphi_{\lambda_{1}}(0) \exp \left[i\left(-\xi_{1}+i \eta_{1}\right) \cdot P\right] \ldots\right. \\
& \left.\ldots \varphi_{\lambda_{n-1}}(0) \exp \left[i\left(-\xi_{n-1}+i \eta_{n-1}\right) \cdot P\right] \varphi_{\lambda_{n}}(0) \Omega\right)
\end{aligned}
$$

is well defined for $\varepsilon>0, \varepsilon$-independent and holomorphic in the tube. The bounds (4.17) and (4.18), and standard lore on Laplace transforms [24] imply $W$ is the Laplace transform of a tempered distribution. Now consider the integral

$$
\int d x F(x) e^{-t H} \varphi_{\lambda}(x) e^{-t H}, \quad \lambda \in\left(-\frac{1}{2}, \frac{1}{2}\right), \quad t>0,
$$

where $F \in S\left(\mathbb{R}^{2}\right)$. In view of the above this is a well-defined Riemann integral in the strong topology, which defines a bounded operator on $\mathscr{F}_{a}(\mathscr{H})$. Assuming in addition supp $\hat{F}$ is compact, this operator equals the operator $e^{-t H} \varphi_{\lambda}(F) e^{-t H}$ on $\mathscr{D}_{\infty}$, with $\varphi_{\lambda}(F)$ defined in Sect. 4A (since the operators coincide as forms on $\mathscr{D}_{\text {at }}$ ). Thus we may write

$$
\begin{aligned}
\hat{\mathscr{W}}\left(\hat{F}_{1}, \ldots, \hat{F}_{n}\right) & =\lim _{t \rightarrow 0}\left(\Omega, \varphi_{\lambda_{1}}\left(F_{1}\right) e^{-t H} \varphi_{\lambda_{2}}\left(F_{2}\right) \ldots e^{-t H} \varphi_{\lambda_{n}}\left(F_{n}\right) \Omega\right) \\
& =\lim _{t \rightarrow 0} \int d x_{1} \ldots d x_{n} F_{1}\left(x_{1}\right) \ldots F_{n}\left(x_{n}\right) W\left(x_{1}-x_{2}-i t \eta, \ldots, x_{n-1}-x_{n}-i t \eta\right),
\end{aligned}
$$

where $\eta \equiv(1,0)$. Using again well-known results on Laplace transforms [24], the claim follows from this.

Assume that $\hat{F}_{i}^{n} \in C_{0}^{\infty}\left(\mathbb{R}^{2}\right) \rightarrow \hat{F}_{i} \in S\left(\mathbb{R}^{2}\right)$ in $S\left(\mathbb{R}^{2}\right)$ for $n \rightarrow \infty, i=1, \ldots, N$. Then the lemma just proved implies that the vectors

$$
\prod_{i=1}^{N} \varphi_{\lambda_{i}}\left(F_{i}^{n}\right) \Omega, \quad \lambda_{i} \in\left(-\frac{1}{2}, \frac{1}{2}\right),
$$

form a Cauchy sequence and therefore have a strong limit for $n \rightarrow \infty$. We denote the subspace of finite linear combinations of such limits by $D$. Another application 
of temperedness shows that the bounded operator (4.21), where $F \in S\left(\mathbb{R}^{2}\right)$, acting on a vector $\psi \in D$ gives rise to a vector $\zeta_{t}$ with a strong limit $\zeta$ for $t \rightarrow 0$. Thus the operator $\varphi_{\lambda}(F)$ defined by putting $\varphi_{\lambda}(F) \psi=\zeta$ is well defined on $D$. A little reflection also shows that:

i) $\varphi_{\lambda}(F)$ leaves $D$ invariant;

ii) the limit of (4.23) equals $\prod_{i=1}^{N} \varphi_{\lambda_{i}}\left(F_{i}\right) \Omega$;

iii)

$$
\varphi_{\lambda}(F)^{*} D=\varphi_{-\lambda}(\bar{F})
$$

iv) $\mathscr{U}(a, \Lambda)$ leaves $D$ invariant;

v)

$$
\mathscr{U}(a, \Lambda) \varphi_{\lambda}(F) \mathscr{U}(a, \Lambda)^{*}=\varphi_{\lambda}\left(F^{a, \Lambda}\right) \text { on } D .
$$

We can now state the next lemma, which will be proved in Sect. 4D.

Lemma 4.3 (Locality). Let $F, G \in S\left(\mathbb{R}^{2}\right)$ with $\operatorname{supp} F$ and supp $G$ spacelike separated. Then

$$
\left[\varphi_{\lambda}(F), \varphi_{\lambda^{\prime}}(G)\right]_{-} \psi=0, \quad \forall \psi \in D, \quad \forall \lambda, \lambda^{\prime} \in\left(-\frac{1}{2}, \frac{1}{2}\right) .
$$

Consider now the subspace of finite linear combinations of strong limits of vectors of the form (4.23), where $\lambda_{i}$ equals either $\lambda$ or $-\lambda, i=1, \ldots, N$. We denote this subspace by $D_{\lambda}$ and its closure by $\bar{D}_{\lambda}$. Clearly, $\bar{D}_{\lambda}$ is a closed subspace of the charge-zero sector in $\mathscr{F}_{a}(\mathscr{H})$. (We believe that $\bar{D}_{\lambda}$ is equal to this sector, but were unable to prove this.) Thus, the restriction of $\mathscr{U}(a, \Lambda)$ to $\bar{D}_{\lambda}$, denoted $\mathscr{U}_{\lambda}(a, \Lambda)$, does not exhibit isolated mass shells. Therefore the field $\varphi_{\lambda}(x)$ in $\bar{D}_{\lambda}$ cannot describe asymptotic particles, and hence the problem of asymptotic completeness does not arise. But it is obvious from the above that all other Wightman axioms are satisfied, a fact we regard as the main result of this chapter:

Theorem 4.4. The quadruple $\left\langle\bar{D}_{\lambda}, \mathscr{U}_{\lambda}(a, \Lambda), \varphi_{\lambda}(x), D_{\lambda}\right\rangle$, where $\lambda \in(-1 / 2,1 / 2)$, satisfies all Wightman axioms with the exception of asymptotic completeness.

\section{C. $\varphi$-Bounds}

Noting that

$$
(H+1)^{-\alpha} \varphi_{\lambda}(0)(H+1)^{-\alpha}=\Gamma(\alpha)^{-2} \int_{0}^{\infty} d t d u(t u)^{\alpha-1} e^{-t(H+1)} \varphi_{\lambda}(0) e^{-u(H+1)}, \quad \alpha>0,
$$

one sees the inequality (4.17) implies $(H+1)^{-C(\lambda)-\varepsilon} \varphi_{\lambda}(0)(H+1)^{-C(\lambda)-\varepsilon}$ is bounded for any $\varepsilon>0$. [Conversely, boundedness of $(H+1)^{-C(\lambda)} \varphi_{\lambda}(0)(H+1)^{-C(\lambda)}$ is easily seen to imply (4.17).] Thus, for $|\lambda|$ small, (4.17) entails the inequality

$$
\left\|(H+1)^{-1 / 2}\left[\int d x^{1} f\left(x^{1}\right) \varphi_{\lambda}\left(0, x^{1}\right)\right](H+1)^{-1 / 2}\right\| \leqq C \int d x^{1}\left|f\left(x^{1}\right)\right|,
$$

which would be called a $\varphi$-bound in constructive field theory $[42,43]$. We begin this section by proving Lemma 4.1 and then add further comments pertaining to $\varphi$-bounds in the sense of (4.17). 
Proof of Lemma 4.1. Using (4.1) and (3.2) it is readily seen that

$$
\begin{aligned}
e^{-t H} \varphi_{\lambda}(0) e^{-u H}= & \exp \left(Z_{\lambda+-}^{2 t} a^{*} b^{*}\right) \Gamma\left(e^{-t H} Z_{\lambda++} e^{-u H}\right) \\
& \cdot \Gamma\left(e^{-t H} Z_{\lambda-\ldots}^{T} e^{-u H}\right) \exp \left(-Z_{\lambda-+}^{2 u} b a\right)
\end{aligned}
$$

as forms on $\mathscr{D}_{\text {at }}$, where the notation will be clear. To prove the first assertion of the lemma, we therefore need only show the four factors at the right-hand side are bounded operators for $t, u>0$. For the first and the last factor we use Theorem 3.2 and (4.3) to conclude that

$$
\left\|\exp \left(Z_{\lambda+-}^{s} a^{*} b^{*}\right)\right\| \leqq \operatorname{det}\left(1+\left|Z_{\lambda+-}^{s}\right|\right),
$$

and that

$$
\left\|\exp \left(-Z_{\lambda-+}^{s} b a\right)\right\|=\left\|\exp \left(Z_{-\lambda+-}^{s} a^{*} b^{*}\right)\right\| \leqq \operatorname{det}\left(1+\left|Z_{-\lambda+-}^{s}\right|\right) .
$$

Since $Z_{\lambda-+}^{s}$ is trace class for any $s>0$ (as we have seen in Sect. 2B), boundedness of these factors follows.

Now consider the second factors. From the pseudo-unitarity of $Z_{\lambda}$ for $\lambda \in(-1 / 2,1 / 2)$, we obtain

$$
\begin{aligned}
\left\|\Gamma\left(e^{-t H} Z_{\lambda++} e^{-u H}\right)\right\|^{2} & \leqq\left\|\Gamma\left(e^{-u H} Z_{\lambda++}^{*} Z_{\lambda++} e^{-u H}\right)\right\| \\
& =\left\|\Gamma\left(e^{-u H}\left[1+Z_{\lambda-+}{ }^{*} Z_{\lambda-+}\right] e^{-u H}\right)\right\| .
\end{aligned}
$$

Using the relation

$$
0<A \leqq B \Rightarrow 0<\Gamma(A) \leqq \Gamma(B),
$$

whose proof is easy, and noting $Z_{\lambda_{-+}}=-Z_{\lambda_{+-}}$as operators on $L^{2}(d \theta)$, we now get

$$
\begin{aligned}
\left\|\Gamma\left(e^{-t H} Z_{\lambda++} e^{-u H}\right)\right\| & \leqq\left\|\Gamma\left(1+e^{-u H} Z_{\lambda+-} * Z_{\lambda+-} e^{-u H}\right)\right\|^{1 / 2} \\
& =\operatorname{det}^{1 / 2}\left(1+e^{-u H} Z_{\lambda+-}{ }^{*} Z_{\lambda+-} e^{-u H}\right)
\end{aligned}
$$

where we used Theorem 3.1 in the last step. Likewise we obtain [using also $\left.\left\|\Gamma\left(A^{T}\right)\right\|=\|\Gamma(A)\|\right]$,

$$
\left\|\Gamma\left(e^{-t H} Z_{\lambda--}^{T} e^{-u H}\right)\right\| \leqq \operatorname{det}^{1 / 2}\left(1+e^{-t H} Z_{\lambda+-}{ }^{*} Z_{\lambda+-} e^{-t H}\right) .
$$

But from Sect. $2 \mathrm{~B}$ we know that $e^{-s H} Z_{\lambda+-}{ }^{*} Z_{\lambda+-} e^{-s H}$ is trace class for $s>0$, so that these factors are bounded operators as well. This proves the first assertion.

To prove the bound (4.17) we use the inequality $\operatorname{det}(1+|T|) \leqq \exp \left(\|T\|_{1}\right)$ on the four bounds just obtained. We may then use the trace norm bounds (2.42) and (2.43) and the relation

$$
\lim _{s \rightarrow 0} K_{0}(s) / \ln \left(\frac{1}{s}\right)=1
$$

to conclude (4.17) holds true.

Let us comment on the difficulties in extending the bound (4.17) to larger and/or complex $\lambda$, and in proving similar $\varphi$-bounds in related models. Inspection 
of the estimates shows there is no difficulty in extending the bounds on the first and last factor in (4.29) to the strip $S$. It is the $\Gamma$-factors that cause trouble: As soon as $\operatorname{Im} \lambda \neq 0, Z_{\lambda}$ fails to be pseudo-unitary, so that the last step in (4.32) is false. Worse yet, for fixed $\operatorname{Im} \lambda \neq 0$ it is likely that $\Gamma\left(e^{-t H} Z_{\lambda++} e^{-u H}\right)$ (e.g.) is unbounded for $t, u$ small enough. Indeed, from Theorem 3.1 we know that boundedness is equivalent to the positive part of $\left|e^{-t H} Z_{\lambda++} e^{-u H}\right|-1$ being trace class. However, from (2.31) we see that $\left|\hat{Z}_{\lambda++}(y)\right| \rightarrow \exp (\mp \pi \operatorname{Im} \lambda)$ for $y \rightarrow \pm \infty$, so it is plausible that this is not the case. Of course, the condition is trivially satisfied for $t, u$ large enough. This leads to the Wightman functions being analytic in the strip $S$ in a subset of the extended tube that shrinks as $\left|\operatorname{Im} \lambda_{i}\right|$ increases. More generally, a similar situation is readily seen to obtain for $\lambda_{i} \in \mathbb{C}$. But since we see no way of making use of this, we have not pursued this further.

In the case of the fermionic Ising model the role of $Z_{\lambda_{+-}}(\theta)$ is played by the function $Z_{+-}(\theta)=(2 \pi i)^{-1} \operatorname{th} \theta / 2$. The first statement of the lemma can be shown to hold true, but the difficulty here is to prove the norm does not blow up faster than a power. It can be seen this is true if $\operatorname{det}\left(1+\left|Z_{+-}^{t}\right|\right)$ has this property, but so far we have only proved the determinant is $O\left((1 / t)^{c \ln (\ln (1 / t))}\right)$. Of course, in this case temperedness is known from $[26,28]$, but note these results do not imply the validity of the analog of Lemma 4.1.

For the bosonic Federbush model the analog of $Z_{\lambda}$ is unitary, so that the $\Gamma$-factors are trivially bounded. The first and last factors can be shown to be bounded for $t, u$ large enough, using number operator estimates. But they are most likely unbounded for $t, u$ small. Of course, this does not preclude that the product of the four factors may be bounded for any $t, u>0$; this is another open problem.

\section{Locality}

We begin this section by proving the locality lemma, and then add some comments on the cutoff fields employed in the proof.

Proof of Lemma 4.3. In the following, the symbol $\chi$ will be used to denote finite linear combinations of vectors of the form $\prod_{i=1}^{N} \varphi_{\lambda_{i}}\left(F_{i}\right) \Omega$, where $N \geqq 0$, $\lambda_{i} \in(-1 / 2,1 / 2)$ and $\hat{F}_{i} \in C_{0}^{\infty}$. Clearly, such vectors are dense in $D$. Thus we need only show that the function

$$
L\left(G, \lambda^{\prime} ; F, \lambda\right) \equiv\left(\chi_{1}, \varphi_{\lambda^{\prime}}(G) \varphi_{\lambda}(F) \chi_{2}\right), \quad \lambda, \lambda^{\prime} \in\left(-\frac{1}{2}, \frac{1}{2}\right)
$$

where $F$ and $G$ are $C_{0}^{\infty}$-functions with $\operatorname{supp} F$ and supp $G$ spacelike separated, satisfies

$$
L\left(F, \lambda ; G, \lambda^{\prime}\right)=L\left(G, \lambda^{\prime} ; F, \lambda\right), \quad \forall \lambda, \lambda^{\prime} \in\left(-\frac{1}{2}, \frac{1}{2}\right)
$$

We claim this is a consequence of the relation

$$
\begin{aligned}
& \left(e^{-\tau H} \chi_{1}, \varphi_{\lambda}(F) \varphi_{\lambda^{\prime}}(G) \chi_{2}\right) \\
& \quad=\left(e^{-\tau H} \chi_{1}, \varphi_{\lambda^{\prime}}(G) \varphi_{\lambda}(F) \chi_{2}\right), \quad \forall \tau>0, \quad \forall \lambda, \lambda^{\prime} \in(-\delta, \delta),
\end{aligned}
$$


where $\delta$ is a positive number smaller than $1 / 2$. We shall now prove this claim and then prove (4.39).

First, from (4.39) it follows by taking $\tau \rightarrow 0$ that (4.38) holds for any $\lambda, \lambda^{\prime} \in(-\delta, \delta)$. We now assert $L$ has an analytic continuation in $\lambda$ and in $\lambda^{\prime}$ to the strip $S$. Clearly, from this (4.38) follows. To prove the assertion, it suffices to show that a vector of the form

$$
\varphi_{\lambda}(D) \chi, \quad \lambda \in\left(-\frac{1}{2}, \frac{1}{2}\right), \quad D \in C_{0}^{\infty},
$$

has a strongly holomorphic extension into $S$. [Indeed, if the extension is denoted $\psi_{\lambda}$, then $\psi_{\bar{\lambda}}$ is an anti-holomorphic extension. Now combine this with (4.24).] Denoting the projection on the vacuum by $P_{\Omega}$, it is clear $P_{\Omega} \varphi_{\lambda}(D) \chi$ has a strongly holomorphic extension to $\mathbb{C}$, so we need only prove the extension property for $\left(1-P_{\Omega}\right) \varphi_{\lambda}(D) \chi$. Now recall that by Stone's theorem, covariance and temperedness the polynomial domain consists of $C^{\infty}(H)$-vectors. In particular, $H$ is well defined on $\varphi_{\lambda}(D) \chi$ and we may use these three tools to trade $H$ for time derivatives of $D$ and the test functions occurring in $\chi$. Since we can write

$$
\left(1-P_{\Omega}\right) \varphi_{\lambda}(D) \chi=H^{-N}\left(1-P_{\Omega}\right) H^{N} \varphi_{\lambda}(D) \chi
$$

this argument implies the right-hand side may be written as a finite linear combination of vectors of the form

$$
H^{-N}\left(1-P_{\Omega}\right) \varphi_{\lambda}(E) \chi, \quad \lambda \in\left(-\frac{1}{2}, \frac{1}{2}\right), \quad E \in C_{0}^{\infty} .
$$

(Recall our convention concerning the symbol $\chi$.) Thus it is sufficient to show that such vectors have a strongly analytic extension into the rectangle $R_{n}$ with corners $-1 / 2+1 / n \pm i n, 1 / 2-1 / n \pm i n$ for any integer $n$, provided $N=N_{n}$ is chosen large enough.

In order to prove this, consider the vector

$$
\psi(\lambda, x, t) \equiv\left(1-P_{\Omega}\right) e^{-t H} \varphi_{\lambda}(x) \chi, \quad t>0, \quad \lambda \in\left(-\frac{1}{2}, \frac{1}{2}\right) .
$$

Using the factorization (4.29), we may write $\psi$ as

$$
e^{i P \cdot x} e^{-1 / 2 t H}\left(1-P_{\Omega}\right) \exp \left(Z_{\lambda+-}^{t} a^{*} b^{*}\right) e^{-1 / 2 t H} \zeta(\lambda, x) .
$$

Now from the fact that $\chi \in \mathscr{D}_{\infty}$, it readily follows $\zeta$ has a strongly analytic continuation into $S$, which is moreover strongly continuous in $x$. Also, from Sect. 2B we know that for fixed $t>0 Z_{\lambda+-}^{t}$ has a $\|\cdot\|_{1}$-analytic continuation into $S$. Thus, from Theorem 3.3 it follows that $\exp \left(Z_{\lambda+-}^{t} a^{*} b^{*}\right)$ has a norm-analytic extension into $S$. Furthermore, combining the bound (3.14) with the bounds (2.43)(2.46) we infer that

$$
\begin{aligned}
& \left\|\left[\exp \left(Z_{\lambda+-}^{t} a^{*} b^{*}\right)-\exp \left(Z_{\lambda_{0}+-}^{t} a^{*} b^{*}\right)\right]\left(\lambda-\lambda_{0}\right)^{-1}-Z_{\lambda_{0}+-}^{t} a^{*} b^{*} \exp \left(Z_{\lambda_{0}+-}^{t} a^{*} b^{*}\right)\right\| \\
& \quad \leqq \exp \left[C\left(\lambda_{0}\right) K_{0}(t)\right]\left(C\left(\lambda, \lambda_{0}\right) K_{0}(t)+C\left(\lambda, \lambda_{0}\right) K_{0}(t) \exp \left[C\left(\lambda, \lambda_{0}\right) K_{0}(t)\right]\right) \\
& \quad \leqq C\left(\lambda, \lambda_{0}\right)\left(\frac{1}{t}\right)^{C\left(\lambda_{0}\right)+C\left(\lambda, \lambda_{0}\right)}
\end{aligned}
$$


where we used (4.36). (Also, recall our convention at the end of Chap.1.) Telescoping and estimating in the obvious way, it follows from the above that $\psi(\lambda, x, t)$ has a strongly analytic extension into $S$, which is strongly continuous in $(x, t)$ on $\mathbb{R}^{2} \times \mathbb{R}^{+}$, and which satisfies the bounds

$$
\begin{gathered}
\|\psi(\lambda, x, t)\| \leqq(C+C(\lambda)) e^{-\frac{1}{4} t}\left(\frac{1}{t}\right)^{C(\lambda)}, \\
\left\|\psi^{\prime}(\lambda, x, t)\right\| \leqq(C+C(\lambda))\left(K_{0}(t)+1\right) e^{-\frac{1}{4} t}\left(\frac{1}{t}\right)^{C(\lambda)}, \\
\left\|\left[\psi(\lambda, x, t)-\psi\left(\lambda_{0}, x, t\right)\right]\left(\lambda-\lambda_{0}\right)^{-1}-\psi^{\prime}\left(\lambda_{0}, x, t\right)\right\| \\
\leqq C\left(\lambda, \lambda_{0}\right) e^{-\frac{1}{4} t}\left(\frac{1}{t}\right)^{C\left(\lambda_{0}\right)+C\left(\lambda, \lambda_{0}\right)} .
\end{gathered}
$$

[Use (3.10) and (2.47) to see (4.47) holds true.] Since $C(\lambda)$ is continuous on $S$, it is bounded above by $\alpha_{n}<\infty$ on the rectangle $R_{n}$. Fixing $N>\alpha_{n}$, we infer from (4.46) and (4.47) that the strong Riemann integrals

$$
\Gamma(N)^{-1} \int_{0}^{\infty} d t t^{N-1} \int d x E(x) \psi(\lambda, x, t)
$$

and

$$
\Gamma(N)^{-1} \int_{0}^{\infty} d t t^{N-1} \int d x E(x) \psi^{\prime}(\lambda, x, t)
$$

converge for $\lambda \in R_{n}$. Also, from (4.48) and dominated convergence, we conclude the vector defined by (4.49) is strongly analytic and has (4.50) as its derivative. Since it is equal to (4.42) for $\lambda \in(-1 / 2+1 / n, 1 / 2-1 / n)$, our assertion holds true.

We are now reduced to proving (4.39). To this end we shall make use of the cutoff field operators $\varphi_{\lambda, \varepsilon}(x)$ introduced in Chap. 3. However, we shall also need three relations involving the two-point function of the field $\varphi_{\lambda}(x)$,

$$
W_{\lambda, \lambda^{\prime}}(x-y) \equiv\left[\varphi_{-\lambda}(x) \Omega, \varphi_{\lambda^{\prime}}(y) \Omega\right] .
$$

(Henceforth, $[\cdot]$ denotes tempered distributions.) We begin by deriving these relations. First, we know already $W$ is the boundary value of a function holomorphic in the tube and invariant under real Lorentz transformations. It follows from this by standard arguments [24] that $W(x)$ is real-analytic in the spacelike region and that

$$
W_{\lambda, \lambda^{\prime}}(x)=W_{\lambda, \lambda^{\prime}}(-x), \quad x^{2}<0 .
$$

We now claim that

$$
W_{\lambda, \lambda^{\prime}}(x)=W_{\lambda^{\prime}, \lambda}(x), \quad \forall x \in \mathbb{R}^{2}
$$

By analyticity it suffices to show this for the Schwinger points. Rotational invariance of the two-point Schwinger function in turn implies that (4.53) follows 
from

$$
W_{\lambda, \lambda^{\prime}}(-i t, 0)=W_{\lambda^{\prime}, \lambda}(-i t, 0), \quad \forall t>0
$$

But using (3.6) we see (4.54) can also be written

$$
\operatorname{det}\left(1+Z_{-\lambda+-}^{t} Z_{\lambda^{\prime}+-}^{t}\right)=\operatorname{det}\left(1+Z_{-\lambda^{\prime}+-}^{t}{ }^{*} Z_{\lambda+-}^{t}\right), \quad \forall t>0 .
$$

Since $Z_{-\lambda+-^{*}}^{*}=Z_{\lambda+-}^{t}$ as operators on $L^{2}(d \theta)$, (4.55) follows from the relation $\operatorname{det}(1+A B)=\operatorname{det}(1+B A)$. Thus, (4.53) is proved. Combining (4.52) and (4.53) we infer that

$$
\begin{aligned}
{\left[\varphi_{-\lambda}(x) \Omega, \varphi_{\lambda^{\prime}}(y) \Omega\right]=} & {\left[\varphi_{-\lambda^{\prime}}(y) \Omega, \varphi_{\lambda}(x) \Omega\right], \quad \forall \lambda, \lambda^{\prime} \in\left(-\frac{1}{2}, \frac{1}{2}\right), } \\
& \forall x, y \in \mathbb{R}^{2}, x-y \text { spacelike, }
\end{aligned}
$$

which is the first relation we need.

Also, using the complex Lorentz transformation $i \sigma_{1}$, we have for $x, y \in \mathbb{R}^{2}$ with $x-y$ spacelike and $x$ to the right of $y$ (e.g.),

$$
\begin{aligned}
\left(e^{-t H} \varphi_{-\lambda}(x) \Omega, e^{-t H} \varphi_{\lambda^{\prime}}(y) \Omega\right) & =W_{\lambda, \lambda^{\prime}}\left(i\left(y^{1}-x^{1}\right), i\left(y^{0}-x^{0}\right)-2 t\right) \\
& =\operatorname{det}\left(1+T_{\lambda, \lambda^{\prime}}(x, y, t)\right), \quad \forall t \geqq 0,
\end{aligned}
$$

where the argument of $W_{\lambda, \lambda^{\prime}}$ is in the tube (since $x$ is to the right of $y$ ), and where $T_{\lambda, \lambda^{\prime}}$ denotes a trace class operator. (This follows from arguments that will be familiar by now.) Moreover, for $\lambda, \lambda^{\prime} \in(-\gamma, \gamma)$ we have $\left\|T_{\lambda, \lambda^{\prime}}\right\|<1$ by (2.54), so that

$$
\begin{gathered}
\left(e^{-t H} \varphi_{-\lambda}(x) \Omega, e^{-t H} \varphi_{\lambda^{\prime}}(y) \Omega\right) \neq 0, \quad \forall t \geqq 0, \quad \forall \lambda, \lambda^{\prime} \in(-\gamma, \gamma), \\
\forall x, y \in \mathbb{R}^{2}, x-y \text { spacelike, }
\end{gathered}
$$

which is the second relation. Finally, the Wightman function $W_{\lambda, \lambda^{\prime}}$ in (4.57) is analytic for $(x, y, t)$ in the compact region $\operatorname{supp} F \times \operatorname{supp} G \times[0,1 / 4]$. Since it cannot vanish there for $|\lambda|,\left|\lambda^{\prime}\right|<\gamma$, it stays a finite distance away from zero, and therefore we have the relation

$$
\begin{gathered}
F(x) G(y)\left(e^{-t H} \varphi_{-\lambda}(x) \Omega, e^{-t H} \varphi_{\lambda^{\prime}}(y) \Omega\right)^{-1} \frac{t \rightarrow 0}{{\mathbf{S}\left(\mathbb{R}^{4}\right)}^{\prime}} \\
F(x) G(y)\left[\varphi_{-\lambda}(x) \Omega, \varphi_{\lambda^{\prime}}(y) \Omega\right]^{-1}, \quad \lambda, \lambda^{\prime} \in(-\gamma, \gamma),
\end{gathered}
$$

which is the third one.

We are now prepared to embark on the proof of (4.39). In view of (4.56) and the analyticity of the reciprocals for $\lambda, \lambda^{\prime} \in(-\gamma, \gamma)$ we need only show there is a positive $\delta \leqq \gamma$ such that

$$
\begin{gathered}
\int d x d y F(x) G(y)\left(\frac{\left[\varphi_{-\lambda}(x) e^{-\tau H} \chi_{1}, \varphi_{\lambda^{\prime}}(y) \chi_{2}\right]}{\left[\varphi_{-\lambda}(x) \Omega, \varphi_{\lambda^{\prime}}(y) \Omega\right]}-\left(\lambda \leftrightarrow \lambda^{\prime}, x \leftrightarrow y\right)\right) \\
=0, \quad \forall \tau>0, \quad \forall \lambda, \lambda^{\prime} \in(-\delta, \delta) .
\end{gathered}
$$

To prove this let us pick $\bar{\varepsilon} \leqq 1$ so small that (3.33) holds on $\operatorname{supp} F \times \operatorname{supp} G$ when we take $\varepsilon \in(0, \bar{\varepsilon})$. We can then telescope the difference at the left-hand side of $(4.60)$ 
into eight terms, as follows (to unburden the notation we omit the coupling constants and absorb the factor $e^{-\tau H}$ into $\chi_{1}$ henceforth):

$$
\begin{aligned}
& \int d x d y F(x) G(y)\left[\frac{\left[\varphi(x) \chi_{1}, \varphi(y) \chi_{2}\right]}{[\varphi(x) \Omega, \varphi(y) \Omega]}-\frac{\left(e^{-t H} \varphi(x) \chi_{1}, e^{-t H} \varphi(y) \chi_{2}\right)}{\left(e^{-t H} \varphi(x) \Omega, e^{-t H} \varphi(y) \Omega\right)}\right] \\
& \quad+\int d x d y \frac{F(x) G(y)}{\left(e^{-t H} \varphi(x) \Omega, e^{-t H} \varphi(y) \Omega\right)}\left[\left(e^{-t H} \varphi(x) \chi_{1}, e^{-t H} \varphi(y) \chi_{2}\right)\right. \\
& \left.\quad-\left(e^{-t H} \varphi(x) e^{t H} \chi_{1}, e^{-t H} \varphi(y) e^{t H} \chi_{2}\right)\right] \\
& +\int d x d y F(x) G(y)\left[\frac{\left(e^{-t H} \varphi(x) e^{t H} \chi_{1}, e^{-t H} \varphi(y) e^{t H} \chi_{2}\right)}{\left(e^{-t H} \varphi(x) \Omega, e^{-t H} \varphi(y) \Omega\right)}-\left(\varphi \rightarrow \varphi_{\varepsilon}\right)\right] \\
& \quad+\int d x d y F(x) G(y)\left[\frac{\left(e^{-t H} \varphi_{\varepsilon}(x) e^{t H} \chi_{1}, e^{-t H} \varphi_{\varepsilon}(y) e^{t H} \chi_{2}\right)}{\left(e^{-t H} \varphi_{\varepsilon}(x) \Omega, e^{-t H} \varphi_{\varepsilon}(y) \Omega\right)}-\frac{\left(\varphi_{\varepsilon}(x) \chi_{1}, \varphi_{\varepsilon}(y) \chi_{2}\right)}{\left(\varphi_{\varepsilon}(x) \Omega, \varphi_{\varepsilon}(y) \Omega\right)}\right] \\
& \quad-(x \leftrightarrow y) .
\end{aligned}
$$

We shall only estimate the first four terms, since the other four terms have the same structure.

Term (i). Consider the functions

$$
K_{t}(x, y) \equiv F(x) G(y)\left(e^{-t H} \varphi(x) \Omega, e^{-t H} \varphi(y) \Omega\right)^{-1} .
$$

In view of the above, in particular (4.59), these functions are in $S\left(\mathbb{R}^{4}\right)$ and have limit $F(x) G(y)[\varphi(x) \Omega, \varphi(y) \Omega]^{-1} \in S$ for $t \rightarrow 0$, provided $\lambda, \lambda^{\prime} \in(-\gamma, \gamma)$. It follows they form a bounded set in $S$. Telescoping (i) into

$$
\begin{aligned}
& \int d x d y\left[\varphi(x) \chi_{1}, \varphi(y) \chi_{2}\right]\left(K_{0}(x, y)-K_{t}(x, y)\right) \\
& \quad+\int d x d y K_{t}(x, y)\left[\left[\varphi(x) \chi_{1}, \varphi(y) \chi_{2}\right]-\left(e^{-t H} \varphi(x) \chi_{1}, e^{-t H} \varphi(y) \chi_{2}\right)\right],
\end{aligned}
$$

it is clear that the first term vanishes for $t \rightarrow 0$. The distribution in brackets in the second term converges to 0 in $S^{\prime}$ for $t \rightarrow 0$ by Laplace transform lore. Thus, by the uniform boundedness principle, it converges to 0 uniformly on bounded sets in $S$. Hence the second term vanishes too for $t \rightarrow 0$. It follows that term (i) vanishes for $t \rightarrow 0$, provided $|\lambda|,\left|\lambda^{\prime}\right|<\gamma$.

Term (ii). This term can be written

$$
\begin{gathered}
\int d x d y K_{t}(x, y)\left[\left(e^{t H}\left[1-e^{t H}\right] \chi_{1}, e^{-t H} \varphi(x) e^{-2 t H} \varphi(y) \chi_{2}\right)\right. \\
\left.+\left(e^{-t H} \varphi(y) e^{-2 t H} \varphi(x) e^{t H} \chi_{1}, e^{t H}\left[1-e^{t H}\right] \chi_{2}\right)\right] .
\end{gathered}
$$

Consider the first inner product. Doing the integration leads to a right-hand side vector that strongly converges to the vector

$$
\int d x d y K_{0}(x, y) \varphi(x) \varphi(y) \chi_{2}
$$

by the argument given for term (i). The left-hand side vector strongly converges to 0 , implying the first term vanishes for $t \rightarrow 0$. The additional factor $e^{-\tau H}$ in $\chi_{1}$ 
ensures the same argument can be applied to the second term as well, implying term (ii) vanishes for $t \rightarrow 0$, provided $|\lambda|,\left|\lambda^{\prime}\right|<\gamma$.

Term (iii). To handle this term and the next one we make use of the following simple fact:

Assume $F\left(z_{1}, z_{2}\right)$ is antiholomorphic/holomorphic in $z_{1} / z_{2}$ in the region

$$
\left\{z_{1}, z_{2} \in \mathbb{C} \mid z_{j}=a_{j}+i t_{j}, a_{j} \in O_{j} \subset \mathbb{R}, O_{j} \text { open, } t_{j}>0, j=1,2\right\} \text {. }
$$

Then one has for $t, t^{\prime}>0$ and $a_{j} \in O_{j}$,

$$
\begin{aligned}
& F\left(a_{1}+i t, a_{2}+i t\right)=F\left(a_{1}+i t^{\prime}, a_{2}+i t^{\prime}\right)+i \partial_{a_{1}} \int_{t}^{t^{\prime}} d s F\left(a_{1}+i s, a_{2}+i t^{\prime}\right) \\
& -i \partial_{a_{2}} \int_{t}^{t^{\prime}} d s^{\prime} F\left(a_{1}+i t^{\prime}, a_{2}+i s^{\prime}\right)+\partial_{a_{1}} \partial_{a_{2}} \int_{t}^{t^{\prime}} d s \int_{t}^{t^{\prime}} d s^{\prime} F\left(a_{1}+i s, a_{2}+i s^{\prime}\right) .
\end{aligned}
$$

[Indeed, by dominated convergence we may interchange the differentiations and integrations. Using the Cauchy-Riemann equations and integrating by parts it follows that (4.67) holds.]

To apply this to (iii) we note that, e.g.,

$$
e^{-t H} \varphi(x) e^{t H}=\exp \left[i H\left(x^{0}+i t\right)\right] \varphi\left(0, x^{1}\right) \exp \left[-i H\left(x^{0}+i t\right)\right]
$$

on $\mathscr{D}_{\infty}$. From this and the relations (3.31) and (4.58) it is straightforward to conclude that the assumptions are satisfied for the two terms inside the square brackets, when $(x, y)$ belongs to $\operatorname{supp} F \times \operatorname{supp} G$. Hence we may write (iii) as

$$
\begin{aligned}
& \int d x d y F(x) G(y) D_{\varepsilon}\left(x, y, \frac{1}{4}, \frac{1}{4}\right)-i \int d x d y\left(\partial_{0} F\right)(x) G(y) \int_{t}^{1 / 4} d s D_{\varepsilon}\left(x, y, s, \frac{1}{4}\right) \\
& +i \int d x d y F(x)\left(\partial_{0} G\right)(y) \int_{t}^{1 / 4} d s^{\prime} D_{\varepsilon}\left(x, y, \frac{1}{4}, s^{\prime}\right) \\
& +\int d x d y\left(\partial_{0} F\right)(x)\left(\partial_{0} G\right)(y) \int_{t}^{1 / 4} d s \int_{t}^{1 / 4} d s^{\prime} D_{\varepsilon}\left(x, y, s, s^{\prime}\right),
\end{aligned}
$$

where we have introduced the difference function

$$
D_{\varepsilon}\left(x, y, s, s^{\prime}\right) \equiv \frac{\left(e^{-s H} \varphi(x) e^{s H} \chi_{1}, e^{-s^{\prime} H} \varphi(y) e^{s^{\prime} H} \chi_{2}\right)}{\left(e^{-s H} \varphi(x) \Omega, e^{-s^{\prime} H} \varphi(y) \Omega\right)}-\left(\varphi \rightarrow \varphi_{\varepsilon}\right) .
$$

We now claim there exists a $\delta_{1} \leqq \gamma$ so that for $\lambda, \lambda^{\prime} \in\left(-\delta_{1}, \delta_{1}\right)$ one has

$$
\left|D_{\varepsilon}\left(x, y, s, s^{\prime}\right)\right| \leqq C\left(\frac{1}{s s^{\prime}}\right)^{1 / 4}
$$

and

$$
\lim _{\varepsilon \rightarrow 0} D_{\varepsilon}\left(x, y, s, s^{\prime}\right)=0
$$


for any $(x, y) \in \operatorname{supp} F \times \operatorname{supp} G$ and $s, s^{\prime} \in\left(0, \frac{1}{4}\right]$. Taking this for granted, it follows from (4.69) and dominated convergence that (iii) vanishes for $\varepsilon \rightarrow 0$, uniformly in $t$. To prove (4.71) and (4.72) we first note that

$$
\begin{aligned}
\left|D_{\varepsilon}\left(x, y, s, s^{\prime}\right)\right| \leqq & \frac{\left|\left(e^{-s H} \varphi(x) e^{s H} \chi_{1}, e^{-s^{\prime} H} \varphi(y) e^{s^{\prime} H} \chi_{2}\right)-\left(\varphi \rightarrow \varphi_{\varepsilon}\right)\right|}{\left|\left(e^{-s H} \varphi(x) \Omega, e^{-s^{\prime} H} \varphi(y) \Omega\right)\right|} \\
& +\frac{\left|\left(e^{-s H} \varphi_{\varepsilon}(x) e^{s H} \chi_{1}, e^{-s^{\prime} H} \varphi_{\varepsilon}(y) e^{s^{\prime} H} \chi_{2}\right)\right|}{\left|\left(e^{-s H} \varphi(x) \Omega, e^{-s^{\prime} H} \varphi(y) \Omega\right)\left(e^{-s H} \varphi_{\varepsilon}(x) \Omega, e^{-s^{\prime} H} \varphi_{\varepsilon}(y) \Omega\right)\right|} . \\
& \cdot\left|\left(e^{-s H} \varphi(x) \Omega, e^{-s^{\prime} H} \varphi(y) \Omega\right)-\left(\varphi \rightarrow \varphi_{\varepsilon}\right)\right| .
\end{aligned}
$$

Consider now the first term. We claim it satisfies a bound of the form (4.71) for $\lambda, \lambda^{\prime}$ small enough and vanishes for $\varepsilon \rightarrow 0$. Since we know already the denominator stays a finite distance away from 0 as $(x, y)$ and $s, s^{\prime}$ vary over $\operatorname{supp} F \times \operatorname{supp} G$ and $\left[0, \frac{1}{4}\right]$ respectively, we need only prove this claim for the numerator. To this purpose we use the Schwarz inequality to conclude that

$$
\begin{aligned}
& \left|\left(e^{-s H} \varphi(x) e^{s H} \chi_{1}, e^{-s^{\prime} H} \varphi(y) e^{s^{\prime} H} \chi_{2}\right)-\left(\varphi \rightarrow \varphi_{\varepsilon}\right)\right| \\
& \leqq \\
& \quad+\left\|e^{-s H}\left(\varphi(x)-\varphi_{\varepsilon}(x)\right) e^{s H} \chi_{1}\right\|\left\|e^{-s^{\prime} H} \varphi(y) e^{s^{\prime} H} \chi_{2}\right\| \\
& \quad+\left\|e^{-s H} \varphi_{\varepsilon}(x) e^{s H} \chi_{1}\right\|\left\|e^{-s^{\prime} H}\left(\varphi(y)-\varphi_{\varepsilon}(y)\right) e^{s^{\prime} H} \chi_{2}\right\| .
\end{aligned}
$$

But using Lemma 2.6 we conclude in a by now familiar fashion that

$$
\begin{aligned}
\left\|e^{-t H} \varphi_{\lambda, \varepsilon}(x)_{\text {p.c. }}\right\| & \leqq \exp \left(\left\|Z_{\lambda, \varepsilon+-}^{t}\right\|_{1}\right) \\
& \leqq C\left(\frac{1}{t}\right)^{C(\lambda)}, \quad \forall t \in\left(0, \frac{1}{4}\right],
\end{aligned}
$$

where p.c. denotes the pure creation part. Likewise, (2.43) entails

$$
\left\|e^{-t H} \varphi_{\lambda}(x)_{\text {p.c. }}\right\| \leqq C\left(\frac{1}{t}\right)^{C(\lambda)}, \quad \forall t \in\left(0, \frac{1}{4}\right] .
$$

Since $C(\lambda)$ vanishes for $\lambda \rightarrow 0$, it follows that the right-hand side of (4.74) is $O\left(\left(s s^{\prime}\right)^{-1 / 4}\right)$ for $\lambda, \lambda^{\prime}$ small enough. Moreover, from Lemma 2.5 and Theorem 3.2 we conclude that

$$
\lim _{\varepsilon \rightarrow 0}\left\|e^{-t H}\left[\varphi_{\lambda, \varepsilon}(x)_{\text {p.c. }}-\varphi_{\lambda}(x)_{\text {p.c. }}\right]\right\|=0, \quad \forall t>0 .
$$

Using also (2.53) and the fact that $\chi_{i} \in \mathscr{D}_{\infty}$, it then easily follows the right-hand side of (4.74) vanishes for $\varepsilon \rightarrow 0$, proving the claim. The second term at the right-hand side of (4.73) can be handled in a similar fashion, except that we now also need the estimate (3.32) to control the denominator. Thus, term (iii) vanishes for $\varepsilon \rightarrow 0$, uniformly in $t$, provided $|\lambda|,\left|\lambda^{\prime}\right|<\delta_{1}$.

Term (iv). Clearly, (3.31) implies the remainder function

$$
R_{\varepsilon}\left(x, y, s, s^{\prime}\right) \equiv \frac{\left(e^{-s H} \varphi_{\varepsilon}(x) e^{s H} \chi_{1}, e^{-s^{\prime} H} \varphi_{\varepsilon}(y) e^{s^{\prime} H} \chi_{2}\right)}{\left(e^{-s H} \varphi_{\varepsilon}(x) \Omega, e^{-s^{\prime} H} \varphi_{\varepsilon}(y) \Omega\right)}
$$


satisfies the assumptions on the function $F$ occurring in (4.67) for any $x, y \in \mathbb{R}^{2}$. If we smear $R_{\varepsilon}(x, y, t, t)$ with $F(x) G(y)$ and then use (4.67) and integration by parts, we see that $t^{\prime}$ may be taken to 0 , which implies (iv) can be written

$$
\begin{gathered}
i \int d x d y\left(\partial_{0} F\right)(x) G(y) \int_{0}^{t} d s R_{\varepsilon}(x, y, s, 0)-i \int d x d y F(x)\left(\partial_{0} G\right)(y) \int_{0}^{t} d s^{\prime} R_{\varepsilon}\left(x, y, 0, s^{\prime}\right) \\
+\int d x d y\left(\partial_{0} F\right)(x)\left(\partial_{0} G\right)(y) \int_{0}^{t} d s \int_{0}^{t} d s^{\prime} R_{\varepsilon}\left(x, y, s, s^{\prime}\right)
\end{gathered}
$$

Arguing now as for (iii) and using the bounds (3.32) and (4.75), it follows there exists a $\delta_{2} \leqq \gamma$ so that for $\lambda, \lambda^{\prime} \in\left(-\delta_{2}, \delta_{2}\right) R_{\varepsilon}$ satisfies

$$
\begin{aligned}
& \left|R_{\varepsilon}\left(x, y, s, s^{\prime}\right)\right| \leqq C\left(\frac{1}{s s^{\prime}}\right)^{1 / 4} \\
& \left|R_{\varepsilon}(x, y, s, 0)\right| \leqq C\left(\frac{1}{s}\right)^{1 / 4} \\
& \left|R_{\varepsilon}\left(x, y, 0, s^{\prime}\right)\right| \leqq C\left(\frac{1}{s^{\prime}}\right)^{1 / 4}
\end{aligned}
$$

for any $x, y \in \mathbb{R}^{2}$ and $s, s^{\prime} \in\left(0, \frac{1}{4}\right]$. Using this to bound (4.79), it follows term (iv) vanishes for $t \rightarrow 0$, uniformly in $\varepsilon$, provided $|\lambda|,\left|\lambda^{\prime}\right|<\delta_{2}$.

Combining these results we finally conclude that for fixed $|\lambda|,\left|\lambda^{\prime}\right|<\delta \equiv \min$ $\left(\gamma, \delta_{1}, \delta_{2}\right)$ we can make (4.61) arbitrarily small by taking $t$ and $\varepsilon$ small enough. Therefore it follows (4.60) holds true, so that the proof of the lemma is complete.

The reader will have noted that we have made essential, but in a certain sense minimal, use of the cutoff fields $\varphi_{\lambda, \varepsilon}$ in the above proof. This is due to our lack of knowledge as regards their properties for $|\lambda| \geqq \frac{1}{4}$ and more generally $\lambda \in \mathbb{C}$, and as regards the strongest topology in which their Wightman distributions converge to the non-cutoff Wightman distributions. It is for instance clear from Sect. 2C and our remarks in Sect. $4 \mathrm{~A}$ that convergence takes place in $\mathscr{D}^{\prime}$ for any $\lambda \in\left(-\frac{1}{4}, \frac{1}{4}\right)$, and the above techniques can be used to show the cutoff two-point function $\left(\Omega, \varphi_{\lambda, \varepsilon}(x) \varphi_{\lambda^{\prime}, \varepsilon}(y) \Omega\right)$ in fact converges to $\left[\Omega, \varphi_{\lambda}(x) \varphi_{\lambda^{\prime}}(y) \Omega\right]$ in $S^{\prime}$, but they are already not strong enough to conclude this for the three-point function.

As concerns large coupling constants, we have already noted in Sect. 2 C. 3 that the analytic Fredholm theorem implies $\varphi_{\lambda, \varepsilon, c l}(x)_{\ldots}$. has for fixed $\varepsilon$ a bounded inverse for any $\lambda \in \mathbb{R}$ but for a discrete set. Thus cutoff quantum fields $\varphi_{\lambda, \varepsilon}(x)$ of the form $E(Z)$ do exist for non-exceptional $\lambda \in \mathbb{R}$. (On the discrete set the implementer has a more complicated form, cf. Theorem 5.1 in [18].) These fields are nonperiodic in $\lambda$, since $\varphi_{\lambda, \varepsilon, c l}(x)$ is. Is it perhaps true that they converge in $\mathscr{D}^{\prime}$ to the non-periodic fields $\varphi_{\lambda}(x)$, although $\varphi_{\lambda, \varepsilon, c l}(x)$ strongly converges to the periodic operator $\varphi_{\lambda, c l}(x)$ ? If so, this would help resolve a puzzle in the literature we have pointed out previously (cf. [20, pp. 358 and 359]). 


\section{The Field $\psi_{2}$}

\section{A. Preliminaries}

The Federbush model describes two species of charged massive fermions. Correspondingly, one can take the Fock space $\mathscr{F}_{a}\left(\mathscr{H}_{1} \oplus \mathscr{H}_{-1}\right)$ as its arena, where $\mathscr{H}_{s}, s= \pm 1$, are copies of the space $\mathscr{H}=L^{2}(d \theta)^{2}$ of Chap. 2 . The representation of the Poincaré group will be again denoted $\mathscr{U}(a, \Lambda)$ and is defined by

$$
\begin{aligned}
(\mathscr{U}(a, \Lambda) \psi)\left(\theta_{1}, s_{1}, \delta_{1} ; \ldots ; \theta_{N}, s_{N}, \delta_{N}\right) \equiv & \exp \left(i a \cdot \sum_{j=1}^{N} p_{s_{J}}\left(\theta_{j}\right)\right) \\
& \cdot \psi\left(\theta_{1}-\alpha, s_{1}, \delta_{1} ; \ldots ; \theta_{N}-\alpha, s_{N}, \delta_{N}\right),
\end{aligned}
$$

where

$$
p_{s}(\theta) \equiv m(s)(\operatorname{ch} \theta, \operatorname{sh} \theta), \quad m(s)>0 .
$$

Thus, $\mathscr{U}(a, \Lambda)$ (as defined here) corresponds to $\mathscr{U}(m(1) a, \Lambda) \otimes \mathscr{U}(m(-1) a, \Lambda)$ (as defined in Chap. 3) under the canonical isomorphism $\mathscr{F}_{a}\left(\mathscr{H}_{1} \oplus \mathscr{H}_{-1}\right) \simeq \mathscr{F}_{a}\left(\mathscr{H}_{1}\right)$ $\otimes \mathscr{F}_{a}\left(\mathscr{H}_{-1}\right)$. The energy-momentum operator and its zeroth component will be denoted $P$ and $H$, respectively, as before. We now introduce the Federbush field. It is defined by

$$
\psi_{\lambda, s}(x) \equiv \psi_{0, s}(x) \varphi_{\lambda,-s}(x)
$$

Here, $\psi_{0, s}$ is the free Dirac field of mass $m(s)$,

$$
\psi_{0 . s}(x) \equiv\left(\frac{m(s)}{4 \pi}\right)^{1 / 2} \int d \theta\left[c_{s, 1}(\theta)\left(\begin{array}{c}
e^{\frac{1}{2} \theta} \\
e^{-\frac{1}{2} \theta}
\end{array}\right) e^{-i x \cdot p_{s}}+c_{s,-1}^{*}(\theta)\left(\begin{array}{c}
i e^{\frac{1}{2} \theta} \\
-i e^{-\frac{1}{2} \theta}
\end{array}\right) e^{i x \cdot p_{s}}\right],
$$

and $\varphi_{\lambda, s}(x)$ equals the field $\varphi_{-\lambda s}(m(s) x)$ of Chap. 4 on the factor $\mathscr{F}_{a}\left(\mathscr{H}_{s}\right)$. Thus one has, explicitly,

$$
\begin{aligned}
\varphi_{\lambda, s}(x) \equiv & : \exp \left(Z_{-\lambda s+-}^{m(s) x} c_{s, 1}^{*} c_{s,-1}^{*}+\left(Z_{-\lambda s++}^{m(s) x}-1\right) c_{s, 1}^{*} c_{s, 1}\right. \\
& \left.-\left(Z_{-\lambda s--}^{m(s) x}-1\right) c_{s,-1} c_{s,-1}^{*}-Z_{-\lambda s-+}^{m(s) x} c_{s,-1} c_{s, 1}\right):
\end{aligned}
$$

where the kernels are given by (4.2).

The subspaces $\mathscr{D}_{\text {at }}, \mathscr{D}_{N}$, and $\mathscr{D}_{\infty}$ of $\mathscr{F}_{a}(\mathscr{H})$ have obvious counterparts in $\mathscr{F}_{a}\left(\mathscr{H}_{1} \oplus \mathscr{H}_{-1}\right)$ that will be denoted by the same symbols. The field $\psi_{\lambda, s}(x)$ is a quadratic form on $\mathscr{D}_{\text {at }}$ to which the same arguments apply as those in Sect. 4A concerning $\varphi_{\lambda}(x)$. In particular, for any $\lambda \in \mathbb{C}$ and $F \in S\left(\mathbb{R}^{2}\right)^{2}$ with compactly supported Fourier transform $\hat{F}$ one concludes that $\psi_{\lambda, s}(F)$ and its adjoint are welldefined operators on $\mathscr{D}_{\infty}$, which leave $\mathscr{D}_{\infty}$ invariant and satisfy

$$
\psi_{\lambda, s}(F)^{* \uparrow \mathscr{D}_{\infty}}=\psi_{\lambda, s}^{*}(\bar{F})
$$

and

$$
\mathscr{U}(a, \Lambda) \psi_{\lambda, s}(F) \mathscr{U}(a, \Lambda)^{*}=\psi_{\lambda, s}\left(F_{\alpha}^{a, \Lambda}\right) .
$$

Here, $\psi_{\lambda, s}^{*}(x)$ is the (quadratic form) adjoint of $\psi_{\lambda, s}(x)$,

$$
\psi_{\lambda, s}^{*}(x)=\psi_{0, s}^{*}(x) \varphi_{-\bar{\lambda},-s}(x) \text {, }
$$


and

$$
F_{\alpha}^{a, \Lambda}(x) \equiv\left(\begin{array}{cc}
e^{-\frac{1}{2} \alpha} & 0 \\
0 & e^{\frac{1}{2} \alpha}
\end{array}\right) F^{a, \Lambda}(x)
$$

[cf. (4.13)-(4.15)]. Also, the vector in $\mathscr{D}_{\infty}$

$$
\prod_{i=1}^{N} \psi_{\lambda_{l}, s_{t}}^{(*)_{i}}\left(F_{i}\right) \Omega, \hat{F}_{i} \in C_{0}^{\infty}\left(\mathbb{R}^{2}\right)^{2}, \lambda_{i} \in \mathbb{C}, \quad i=1, \ldots, N
$$

depends continuously on $\hat{F}_{i} \in \mathscr{D}\left(\mathbb{R}^{2}\right)^{2}$ and is entire in $\lambda_{i}$ in the strong topology. Here, the notation $(*)_{i}$ indicates that either the field or its adjoint (5.8) occurs, depending on $i$.

\section{B. The Results}

In this section we present the main results of this chapter along the same lines as in Sect. 4B. The proofs can be found in the next section.

Lemma 5.1 (A $\psi$-bound). For any $\lambda \in\left(-\frac{1}{2}, \frac{1}{2}\right)$ and $t>0 \quad e^{-t H} \psi_{\lambda, s, i}(0) e^{-t H}$ is a bounded operator, satisfying

$$
\left\|e^{-t H} \psi_{\lambda, s, i}(0) e^{-t H}\right\|=O\left(\left(\frac{1}{t}\right)^{2 C(\lambda)+\frac{1}{2}}\right), \quad t \rightarrow 0,
$$

where $C(\lambda)$ is the same function as in (4.17) and $i=u / l$ denotes the upper/lower component of $\psi_{\lambda, s}$.

Using this result the discussion following Lemma 4.1 can be repeated with obvious changes. In particular, it easily follows that the distribution in $\mathscr{D}^{\prime}\left(\mathbb{R}^{2 n}\right)^{2^{n}}$ defined by

$$
\hat{\mathscr{W}}\left(\hat{F}_{1}, \ldots, \hat{F}_{n}\right) \equiv\left(\Omega, \prod_{i=1}^{n} \psi_{\lambda_{l}, s_{i}}^{(*)_{i}}\left(F_{i}\right) \Omega\right), \lambda_{i} \in\left(-\frac{1}{2}, \frac{1}{2}\right), \hat{F}_{i} \in \mathscr{D}\left(\mathbb{R}^{2}\right)^{2},
$$

is the restriction of a tempered distribution. Also, if $\hat{F}_{i}^{n} \in C_{0}^{\infty}\left(\mathbb{R}^{2}\right)^{2} \rightarrow \hat{F}_{i} \in S\left(\mathbb{R}^{2}\right)^{2}$ in $S\left(\mathbb{R}^{2}\right)^{2}$ for $n \rightarrow \infty$, then the vectors

$$
\prod_{i=1}^{N} \psi_{\lambda_{i}, s_{i}}^{(*)_{i}}\left(F_{i}^{n}\right) \Omega, \lambda_{i} \in\left(-\frac{1}{2}, \frac{1}{2}\right)
$$

strongly converge for $n \rightarrow \infty$. Again, we denote the subspace of finite linear combinations of such limits by $D$. As in Chap. 4, we can then define the operator $\psi_{\lambda, s}^{(*)}(F)$ on $D$ for any $\lambda \in\left(-\frac{1}{2}, \frac{1}{2}\right)$ and $F \in S\left(\mathbb{R}^{2}\right)^{2}$. The properties i), ., v) have obvious analogs for $\psi_{\lambda, s}$ and $\mathscr{U}(a, \Lambda)$, and therefore need not be spelled out again. We continue with a lemma that details the commutation properties of the Federbush fields.

Lemma 5.2 (Commutation relations). Let $\lambda, \lambda^{\prime} \in\left(-\frac{1}{2}, \frac{1}{2}\right)$ and $F, G \in S\left(\mathbb{R}^{2}\right)^{2}$ with $\operatorname{supp} F$ and $\operatorname{supp} G$ spacelike separated and $\operatorname{supp} F$ to the right of $\operatorname{supp} G$. Then the 
following relations hold on $D$ :

$$
\begin{aligned}
\psi_{\lambda, s}(F) \psi_{\lambda^{\prime}, s}^{*}(G) & =-\psi_{\lambda^{\prime}, s}^{*}(G) \psi_{\lambda, s}(F), \\
\psi_{\lambda, s}(F) \psi_{\lambda^{\prime}, s}(G) & =-\psi_{\lambda^{\prime}, s}(G) \psi_{\lambda, s}(F), \\
\psi_{\lambda, s}(F) \psi_{\lambda^{\prime},-s}^{*}(G) & =-\exp \left(i \pi\left(\lambda^{\prime}-\lambda\right) s\right) \psi_{\lambda^{\prime},-s}^{*}(G) \psi_{\lambda, s}(F), \\
\psi_{\lambda, s}(F) \psi_{\lambda^{\prime},-s}(G) & =-\exp \left(i \pi\left(\lambda-\lambda^{\prime}\right) s\right) \psi_{\lambda^{\prime},-s}(G) \psi_{\lambda, s}(F) .
\end{aligned}
$$

We now define $D_{\lambda} \subset D$ as the subspace of all finite linear combinations of strong limits of vectors of the form (5.13), where all $\lambda_{i}$ are equal to a fixed $\lambda \in\left(-\frac{1}{2}, \frac{1}{2}\right)$. From the above it readily follows the Haag-Ruelle theory [33] is valid for the fields $\psi_{\lambda, s}^{(*)}(F)$ on $D_{\lambda}$. Thus the question of asymptotic completeness makes sense. We shall presently answer this question affirmatively; in fact, we shall prove that the closure of the asymptotic spaces equals $\mathscr{F}_{a}\left(\mathscr{H}_{1} \oplus \mathscr{H}_{-1}\right)$. Thus we have the following theorem, which we regard as the principal result of this paper.

Theorem 5.3. For any $\lambda \in\left(-\frac{1}{2}, \frac{1}{2}\right)$, the quadruple $\left\langle\mathscr{F}_{a}\left(\mathscr{H}_{1} \oplus \mathscr{H}_{-1}\right), \mathscr{U}(a, \Lambda), \psi_{\lambda, s}(x)\right.$, $D_{\lambda}>$ satisfies all Wightman axioms including asymptotic completeness.

\section{The Proofs}

Proof of Lemma 5.1. We first recall that for $f \in L^{2}(d \theta) c_{s, \delta}^{(*)}(f)$ is a bounded operator, satisfying

$$
\left\|c_{s, \delta}^{(*)}(f)\right\|=\|f\|
$$

Using Lemma 4.1 we now conclude that $e^{-t H} \psi_{\lambda, s, i}(0) e^{-t H}$ is a bounded operator of the form $A \otimes B$ on $\mathscr{F}_{a}\left(\mathscr{H}_{1}\right) \otimes \mathscr{F}_{a}\left(\mathscr{H}_{-1}\right)$ for any $t>0$. Noting that for $a>0$

$$
\int d \theta e^{ \pm \theta} e^{-a t \operatorname{ch} \theta}=O\left(\frac{1}{t}\right), \quad t \rightarrow 0,
$$

the bound (5.11) follows by also taking (4.17) into account.

Proof of Lemma 5.2. The proof of this lemma proceeds along the same lines as that of Lemma 4.3, and we shall therefore use the same symbols to denote analogous objects. We shall first prove (5.14). We claim this relation will follow once we show that

$$
\begin{aligned}
\int d x & d y F(x) G(y)\left(\frac{\left[\psi_{\lambda, s}^{*}(x) e^{-\tau H} \chi_{1}, \psi_{\lambda^{\prime}, s}^{*}(y) \chi_{2}\right]}{\left[\varphi_{-\lambda,-s}(x) \Omega, \varphi_{-\lambda^{\prime},-s}(y) \Omega\right]}\right. \\
+ & \left.\frac{\left[\psi_{\lambda^{\prime}, s}(y) e^{-\tau H} \chi_{1}, \psi_{\lambda, s}(x) \chi_{2}\right]}{\left[\varphi_{\lambda^{\prime},-s}(y) \Omega, \varphi_{\lambda,-s}(x) \Omega\right]}\right)=0, \quad \forall \tau>0, \quad \forall \lambda, \lambda^{\prime} \in(-\delta, \delta),
\end{aligned}
$$

where $\delta$ is the same positive number as in the proof of Lemma 4.3 [cf. (4.60)]. [Of course, the $\chi_{i}$ are now finite linear combinations of vectors of the form (5.10) with $\lambda_{i} \in\left(-\frac{1}{2}, \frac{1}{2}\right)$.] The proof of this claim follows from (4.56) and the arguments in the proof of Lemma 4.3 leading to the validity of the analogous claim made below (4.38); the presence of the extra free Dirac field only gives rise to an additional factor $(1 / t)^{1 / 2}$ in (4.46)-(4.48), so that one should take $N>\alpha_{n}+\frac{1}{2}$ in (4.49) and (4.50). 
Notice this factor is only needed for the term containing the creation part of the Dirac field: the form $\int d \theta c_{s, \delta}(\theta) e^{ \pm \frac{1}{2} \theta} e^{-i x \cdot p_{s}}$ defines a bounded operator on $\mathscr{D}_{N}$, since the integral may then the restricted to a bounded region.

To prove the relation (5.20), we first observe it holds if the fields are replaced by the corresponding cutoff fields, provided the cutoff is small enough. [To see this, use (3.33) and (3.44).] Thus we can telescope the left-hand side into eight terms as in the proof of Lemma 4.3. These terms can now be estimated in an analogous way. Therefore we shall only point out the differences. Clearly, (i) and (ii) cause no problem. To handle (iii), note that in the analogs of (4.71), (4.75), and (4.76) the exponents should be increased by $\frac{1}{2}$ to take the Dirac field creation part into account. To conclude that (4.72) holds true, one only needs the additional relation

$$
\lim _{\varepsilon \rightarrow 0}\left\|e^{-t H}\left[\psi_{0, s, \varepsilon}(x)-\psi_{0, s}(x)\right] e^{-t \boldsymbol{H}}\right\|=0, \quad \forall t>0,
$$

which readily follows from (3.40) and (5.18). In (iv) a minor snag is encountered: $R_{\varepsilon}$ now satisfies the bound

$$
\left|R_{\varepsilon}\left(x, y, s, s^{\prime}\right)\right| \leqq C\left(\frac{1}{s s^{\prime}}\right)^{3 / 4}, \quad \forall x, y \in \mathbb{R}^{2}, \quad \forall s, s^{\prime} \in\left(0, \frac{1}{4}\right],
$$

instead of (4.80), which suffices for the third term in (4.79) to vanish as before, but the exponents in the analogs of (4.81) and (4.82) are 5/4, which is not sufficient to handle the first two terms. However, for $t^{\prime}>0$ we may write

$$
\begin{aligned}
& i \int d x d y\left(\partial_{0} F\right)(x) G(y) \int_{t^{\prime}}^{t} d s R_{\varepsilon}\left(x, y, s, t^{\prime}\right) \\
& =\int d x d y\left(\partial_{0} F\right)(x)\left(\partial_{0} G\right)(y) \int_{t^{\prime}}^{t} d s \int_{t^{\prime}}^{1 / 4} d s^{\prime} R_{\varepsilon}\left(x, y, s, s^{\prime}\right) \\
& \quad+i \int d x d y\left(\partial_{0} F\right)(x) G(y) \int_{t^{\prime}}^{t} d s R_{\varepsilon}\left(x, y, s, \frac{1}{4}\right)
\end{aligned}
$$

and if we now take $t^{\prime}$ to 0 we obtain an equation that enables us to replace the first term in (4.79) by two terms that vanish for $t \rightarrow 0$, uniformly in $\varepsilon$, by virtue of the bound (5.22). The second term in (4.79) can be taken care of analogously. It follows that (5.20) holds true, implying (5.14). The proof of (5.15) is similar.

To show that (5.16) holds, we need only prove that

$$
\begin{aligned}
& \int d x d y F(x) G(y)\left(\left[\psi_{\lambda, s}^{*}(x) e^{-\tau H} \chi_{1}, \psi_{\lambda^{\prime},-s}^{*}(y) \chi_{2}\right]\right. \\
& \left.\quad+\exp \left(i \pi\left(\lambda^{\prime}-\lambda\right) s\right)\left[\psi_{\lambda^{\prime},-s}(y) e^{-\tau H} \chi_{1}, \psi_{\lambda, s}(x) \chi_{2}\right]\right)=0, \quad \forall \tau>0, \quad \forall \lambda, \lambda^{\prime} \in(-\delta, \delta),
\end{aligned}
$$

by virtue of the analyticity argument discussed above. A straightforward calculation using (3.45) and the fact that $\operatorname{supp} F$ is to the right of $\operatorname{supp} G$ shows that (5.24) holds true for the cutoff fields, provided the cutoff is small enough. But then we may proceed as before, except that now of course no two-point denominators occur in the analog of (4.61). The eight terms can then be estimated in the same fashion as sketched for (5.20), which results in the validity of $(5.16)$. The proof of (5.17) is similar. 
Proof of Theorem 5.3. We need only prove asymptotic completeness and the relation

$$
\bar{D}_{\lambda}=\mathscr{F}_{a}\left(\mathscr{H}_{1} \oplus \mathscr{H}_{-1}\right)
$$

(cyclicity of the vacuum), all other axioms being obvious from the above. To this end we combine a result of Hepp [34] with a result of ours [21]: Define fields

$$
\psi_{\lambda, s}^{\text {out }} \text { in }(x) \equiv S_{\mp \frac{1}{2} \lambda} \psi_{0, s}(x) S_{ \pm \frac{1}{2} \lambda},
$$

where

$$
\begin{gathered}
\left(S_{\lambda} \psi\right)\left(\theta_{1}, s_{1}, \delta_{1} ; \ldots ; \theta_{N}, S_{N}, \delta_{N}\right) \equiv \exp \left[-i \pi \lambda \sum_{i<j} \varepsilon\left(\theta_{i}-\theta_{j}\right)\left(s_{i}-s_{j}\right) \delta_{i} \delta_{j}\right] \\
\cdot \psi\left(\theta_{1}, s_{1}, \delta_{1} ; \ldots ; \theta_{N}, s_{N}, \delta_{N}\right)
\end{gathered}
$$

This notation is inspired by the following result (Theorem 4.2 in [21]): For any smooth solution $F_{s}(x)$ to the Dirac equation of mass $m(s)$ one has

$$
\begin{aligned}
& \lim _{t \rightarrow \pm \infty} \int d x^{1} \bar{F}_{s}\left(t, x^{1}\right) \cdot\left\langle\psi, \psi_{\lambda, s}\left(t, x^{1}\right) \varphi\right\rangle \\
& \quad=\int d x^{1} \bar{F}_{s}\left(0, x^{1}\right) \cdot\left\langle\psi, \psi_{\lambda, s}^{\text {out }}\left(0, x^{1}\right) \varphi\right\rangle, \quad \forall \psi, \varphi \in \mathscr{D} .
\end{aligned}
$$

Here, $\mathscr{D}$ denotes the subspace of algebraic tensors whose constituent functions are in $C_{0}^{\infty}(\mathbb{R})$. Thus the fields (5.26) are the LSZ asymptotic fields for the quadratic form $\psi_{\lambda, s}(x)$ on $\mathscr{F}_{a}\left(\mathscr{H}_{1} \oplus \mathscr{H}_{-1}\right)$ and $S_{\lambda}$ is the LSZ $S$-matrix. These fields are asymptotically complete by inspection, in the sense that the polynomial domain generated by the fields and their adjoints is clearly dense in $\mathscr{F}_{a}$. Therefore it only remains to be shown that the asymptotic fields of the Haag-Ruelle theory (which exist in $\bar{D}_{\lambda}$, since all of its assumptions are satisfied) coincide with the fields (5.26).

At first glance this seems obvious from Hepp's work, since it is one of his results that the assumptions of the Haag-Ruelle theory entail that the LSZ fields exist and are equal to the Haag-Ruelle fields [34]. However, we do not yet know that the states he uses are the same as those generated by the fields (5.26). Since the LSZ limits of $\psi_{\lambda, s}$ could a priori be different if a different set of states is employed, the result just quoted cannot be used. Recall in this connection that the $L^{1}$-norm of $F_{s}(t, \cdot)$ diverges for $|t| \rightarrow \infty$, so that one cannot conclude equality of the limits from a continuity argument.

However, by using another result of Hepp we can attain our goal, as follows : First, the one-fermion states corresponding to the fields (5.26) and their adjoints are clearly equal to those of the Haag-Ruelle fields. Now we make an induction assumption: Denote the Haag-Ruelle creation operators by $c_{s, \delta}^{* \mathrm{ex}}(\theta)$, ex $=$ in, out, and let

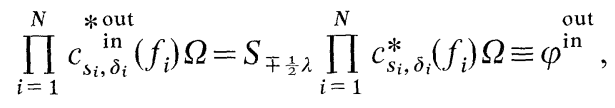


where the $f_{i}$ are in $C_{0}^{\infty}$ and $\operatorname{supp} f_{i}$ is disjoint from supp $f_{i+1}$ and to the left of it, $i=1, \ldots, N-1$. Now consider a negative energy solution of the form

$$
F_{s}(x)=\left(\frac{m(s)}{4 \pi}\right)^{1 / 2} \int d \theta \bar{u}(\theta)\left(\begin{array}{c}
i e^{\frac{1}{2} \theta} \\
-i e^{-\frac{1}{2} \theta}
\end{array}\right) e^{i x \cdot p_{s}},
$$

where $u \in C_{0}^{\infty}$ and $\operatorname{supp} u$ is disjoint from $\operatorname{supp} f_{1}$ and to the left of it. From Theorem 6.2 in [34] we then conclude on one hand that

$$
\underset{s-\lim _{t \rightarrow t^{\mathrm{ex}}}}{ } \psi_{\lambda, s}\left(H, \bar{F}_{s}, t\right) \varphi^{\mathrm{ex}}=c_{s,-1}^{* \mathrm{ex}}(u) \varphi^{\mathrm{ex}},
$$

where

$$
\psi_{\lambda, s}\left(H, \bar{F}_{s}, t\right) \equiv \int d x^{1} \bar{F}_{s}\left(t, x^{1}\right) \cdot \int d y H(y) \psi_{\lambda, s}\left(t-y^{0}, x^{1}-y^{1}\right) .
$$

Here, $H(y)$ is any function whose Fourier transform has the following properties :

(i) it is in $C_{0}^{\infty}\left(\mathbb{R}^{2}\right)$;

(ii) it equals 1 on the set

$$
\left\{p \in \mathbb{R}^{2} \mid p=p_{s}(\theta), \theta \in \operatorname{supp} u\right\} ;
$$

(iii) its support is contained in the set

$$
\left\{p \in \mathbb{R}^{2} \mid p^{0}>0,0<p^{2}<[m(s)+2 m(-s)]^{2}\right\} .
$$

(Note that (i) implies (5.32) is well defined on $\varphi^{\mathrm{ex}}$ in view of our remarks in Sects. $4 \mathrm{~A}$ and $5 \mathrm{~A}$; also use this to verify that (5.32) is of the form considered by Hepp, cf. pp. 195 and 196 in [34].) We now claim that on the other hand

$$
\lim _{t \rightarrow \pm \infty}\left(\psi, \psi_{\lambda, s}\left(H, \bar{F}_{s}, t\right) \varphi^{\substack{\text { out } \\ \text { in }}}\right)=\left(\psi, S_{\mp \frac{1}{2} \lambda} c_{s,-1}^{*}(u) S_{ \pm \frac{1}{2} \lambda} \varphi^{\text {out }} \varphi^{\text {in }}\right), \quad \forall \psi \in \mathscr{D} .
$$

To prove this claim, we first observe that the function $\left\langle\psi, \psi_{\lambda, s}(x) \varphi^{\mathrm{ex}}\right\rangle$ is uniformly bounded for $x \in \mathbb{R}^{2}$. By virtue of Fubini's theorem and covariance we therefore may write

$$
\left(\psi, \psi_{\lambda, s}\left(H, \bar{F}_{s}, t\right) \varphi^{\mathrm{ex}}\right)=\int d y H(y) K(t, y)
$$

where

$$
K(t, y) \equiv \int d x^{1} \bar{F}_{s}\left(t, x^{1}\right) \cdot\left\langle e^{i y \cdot P} \psi, \psi_{\lambda, s}\left(t, x^{1}\right) e^{i y \cdot P} \varphi^{\mathrm{ex}}\right\rangle .
$$

Now it is clear that $e^{i y \cdot P} \psi \in \mathscr{D}$, and one also has $e^{i y \cdot P} \varphi^{\mathrm{ex}} \in \mathscr{D}$, since the operator $S_{\mp \frac{1}{2} \lambda}$ in (5.29) acts as multiplication by a rapidity-independent phase factor due to the restriction on the supports. Therefore our result (5.28) implies that

$$
\lim _{t \rightarrow t^{\mathrm{ex}}} K(t, y)=\int d x^{1} \bar{F}_{s}\left(0, x^{1}\right) \cdot\left\langle\psi, \psi_{\lambda, \mathrm{s}}^{\mathrm{ex}}\left(-y^{0}, x^{1}-y^{1}\right) \varphi^{\mathrm{ex}}\right\rangle .
$$

It is also straightforward to conclude that $K(t, y)$ is uniformly bounded on $\mathbb{R}^{3}$. [Expanding $\varphi_{-\lambda, s}(x)$ in (5.37) leads to a finite sum of terms that are finite products of uniformly bounded functions and a smooth solution of the Klein-Gordon equation due to the free Dirac field factor. Now use the well-known fact that such 
solutions have $L^{\infty}-/ L^{1}$-norms in $x^{1}$ that are $O\left(|t|^{-1 / 2}\right) / O\left(|t|^{1 / 2}\right)$ for $|t| \rightarrow \infty$.] By dominated convergence it therefore follows the limit $t \rightarrow t^{\mathrm{ex}}$ of (5.36) exists, and the ensuing integrals can easily be done. The result of this is the right-hand side of (5.35), which proves our claim.

Combining (5.31) and (5.35) we infer that

$$
\underset{s,-1}{\stackrel{\text { *out }}{\text { in }}(u)} \stackrel{\substack{\text { out } \\ \text { in }}}{c_{\mp \frac{1}{2} \lambda}} c_{s,-1}^{*}(u) S_{ \pm \frac{1}{2} \lambda} \varphi^{\text {out }}
$$

since $\mathscr{D}$ is dense in $\mathscr{F}_{a}$. Using a similar argument for the adjoint field it also follows that (5.39) holds when the charge index -1 is replaced by 1 . Thus we have, replacing $s$ by $s_{0}$ and $u$ by $f_{0}$ and using (5.29),

$$
\prod_{i=0}^{N} c_{s_{i}, \delta_{i}}^{* \text { out }}\left(f_{i}\right) \Omega=S_{\mp \frac{1}{2} \lambda} \prod_{i=0}^{N} c_{s_{i}, \delta_{i}}^{*}\left(f_{i}\right) \Omega .
$$

Hence, it follows by induction that (5.29) holds for any $N$. From this we finally conclude that the fields (5.26) are the asymptotic fields of the Haag-Ruelle theory. This completes the proof of the theorem.

Acknowledgement. The author would like to thank J. Klauder, E. Seiler, and A. S. Wightman for useful discussions.

\section{References}

1. Federbush, P.: A two-dimensional relativistic field theory. Phys. Rev. 121, 1247-1249 (1961)

2. Federbush, P.: Operator equations in two field theory models. Progr. Theor. Phys. 26, 148-150 (1961)

3. Wightman, A.S. : Introduction to some aspects of the relativistic dynamics of quantized fields. In: High energy electromagnetic interactions and field theory. Lévy, M. (ed.), pp. 171-289. New York: Gordon \& Breach 1967

4. Challifour, J.L., Wightman, A.S.: Unpublished manuscript

5. Challifour, J.L.: Time-ordered products in two-dimensional field theories. J. Math. Phys. 9, $1137-1145(1968)$

6. Tapper, M.L. : A possible conflict between renormalized perturbation theory and an exact solution of the two-dimensional Federbush model describing the pseudoscalar interaction of two massive fermions. Nuovo Cimento 25 A, 165-172 (1975)

7. Hählen, P.: Perturbation theory in the Federbush model: induced interaction terms. Nucl. Phys. B116, 491-499 (1976)

8. Butera, P., Enriotti, M.: On the exact solution of the Federbush model. Lett. Nuovo Cimento 16, 545-548 (1976)

9. Singh, L.P.S., Hagen, C.R. : Absence of induced counterterms in the Federbush model. Lett. Nuovo Cimento 21, 529-530 (1978)

10. Schroer, B., Truong, T.T., Weisz, P.: Model study of nonleading mass singularities. I. Ann. Phys. 102, 156-169 (1976)

11. Lehmann, H., Stehr, J.: The Bose field structure associated with a free massive Dirac field in one space dimension (preprint)

12. Sato, M., Miwa, T., Jimbo, M.: Publ. RIMS 14, 223-267 (1978)

13. Sato, M., Miwa, T., Jimbo, M.: Publ. RIMS 15, 201-278 (1979)

14. Sato, M., Miwa, T., Jimbo, M. : Publ. RIMS 15, 577-629 (1979)

15. Sato, M., Miwa, T., Jimbo, M.: Publ. RIMS 15, 871-972 (1979)

16. Sato, M., Miwa, T., Jimbo, M. : Publ. RIMS 16, 531-584 (1980)

17. Sato, M., Miwa, T., Jimbo, M.: Publ. RIMS 17, 137-151 (1981) 
18. Ruijsenaars, S.N.M.: On Bogoliubov transformations for systems of relativistic charged particles. J. Math. Phys. 18, 517-526 (1977)

19. Ruijsenaars, S.N.M.: On Bogoliubov transformations. II. The general case. Ann. Phys. 116, 105-134 (1978)

20. Ruijsenaars, S.N.M. : Integrable quantum field theories and Bogoliubov transformations. Ann. Phys. 132, 328-382 (1981)

21. Ruijsenaars, S.N.M.: Scattering theory for the Federbush, massless Thirring and continuum Ising models. J. Funct. Anal. (to appear)

22. Ruijsenaars, S.N.M.: On the two-point functions of some integrable relativistic quantum field theories. J. Math. Phys. (to appear)

23. Klauder, J.R.: Acta Universitatis Wratislaviensis, No. 519, XVth Winter School of Theoretical Physics in Karpacz, pp. 145-188 (1979)

24. Streater, R.F., Wightman, A.S.: PCT, spin and statistıcs, and all that. New York: Benjamin 1964

25. McCoy, B.M., Tracy, C.A., Wu, T.T.: Two-dimensional Ising model as an exactly soluble relativistic quantum field theory: explicit formulas for $n$-point functions. Phys. Rev. Lett. 38, 793-796 (1977)

26. Palmer, J., Tracy, C.: Adv. Appl. Math. 2, 329-388 (1981)

27. Palmer, J.: Adv. Appl. Math. 2, 290-328 (1981)

28. Schor, R., O'Carroll, M.: The scaling limit and Osterwalder-Schrader axioms for the twodimensional Ising model. Commun. Math. Phys. 84, 153-170 (1982)

29. Schor, R., O'Carroll, M.: The quantum field theory associated with the infinite lattice twodimensional Ising model Commun. Math. Phys. 84, 153-170 (1982)

30. O'Carroll, M., Schor, R.: The correlation functions and scaling limit of the two-dimensional Ising model (preprint)

31. Glimm, J., Jaffe, A. : A remark on the existence of $\varphi_{4}^{4}$. Phys. Rev. Lett. 33, 440-442 (1974)

32. Newman, C.: Z. Wahrsch. Verw. Geb. 33, 75-93 (1975)

33. Jost, R.: The general theory of quantized fields. Am. Math. Soc. Providence 1965

34. Hepp, K.: On the connection between Wightman and LSZ quantum field theory. In: Axiomatic field theory. Chretien, M., Deser, S. (eds.), pp. 135-246. New York: Gordon \& Breach 1966

35. Jaffe, A.M.: High-energy behavior in quantum field theory. I. Strictly localizable fields. Phys. Rev. 158, 1454-1461 (1967)

36. Constantinescu, F.: Distributions and their applications in physics. Oxford: Pergamon Press 1980

37. Carey, A.L., Hurst, C.A., O'Brien, D.M.: Automorphisms of the canonical anticommutation relations and index theory (preprint)

38. Ruijsenaars, S.N.M.: Charged particles in external fields. I. Classical theory. J. Math. Phys. 18, $720-737$ (1977)

39. Ruijsenaars, S.N.M.: Charged particles in external fields. II. The quantized Dirac and KleinGordon theories. Commun. Math. Phys. 52, 267-294 (1977)

40. Raina, A.K., Wanders, G.: The gauge transformations of the Schwinger model. Ann. Phys. 132, 404-426 (1981)

41. Simon, B.: Trace ideals and their applications. Cambridge: Cambridge University Press 1979

42. Simon, B.: The $P(\varphi)_{2}$ Euclidean (quantum) field theory. Princeton: Princeton University Press 1974

43. Glimm, J., Jaffe, A.: Quantum physics. Berlin, Heidelberg, New York: Springer 1981

44. Palmer, J., Tracy, C.: Two dimensional Ising correlations: the SMJ analysis (preprint)

Communicated by K. Osterwalder

Received April 7, 1982 